FTUV-05-0602

\title{
Intrinsic CPT violation and decoherence for entangled neutral mesons
}

\author{
J. Bernabéu ${ }^{a}$, N.E. Mavromatos ${ }^{b}$, J. Papavassiliou $^{a}$, and A. Waldron-Lauda ${ }^{b}$ \\ ${ }^{a}$ Departamento de Física Teórica and IFIC, \\ Centro Mixto, Universidad de Valencia-CSIC, \\ E-46100, Burjassot, Valencia, Spain. \\ ${ }^{b}$ King's College London, University of London, \\ Department of Physics, Strand WC2R 2LS, London, U.K.
}

(Dated: November 18, 2018)

\begin{abstract}
We present a combined treatment of quantum-gravity-induced decoherence and intrinsic CPT violation in entangled neutral-Kaon states. Our analysis takes into consideration two types of effects: first, those associated with the loss of particle-antiparticle identity, as a result of the illdefined nature of the CPT operator, and second, effects due to the non-unitary evolution of the Kaons in the space-time foam. By studying a variety of $\phi$-factory observables, involving identical as well as general final states, we derive analytical expressions, to leading order in the associated CPT violating parameters, for double-decay rates and their time-integrated counterparts. Our analysis shows that the various types of the aforementioned effects may be disentangled through judicious combinations of appropriate observables in a $\phi$ factory.
\end{abstract}

PACS numbers: 11.30.Er; 13.25.Es; 03.65.Ud 


\section{INTRODUCTION}

To date, the Special Theory of Relativity, the established theory of flat space-time physics based on Lorentz symmetry, is very well tested. In fact, during the current year, it completes a century of enormous success, having passed very stringent and diverse experimental tests. On the other hand, a quantum theory of Gravity, that is, a consistent quantized version of Einstein's General Relativity, still eludes us. This may be partially attributed to the lack of any concrete observational evidence on the structure of space-time at the characteristic scale of quantum gravity (QG), the Planck mass-scale $M_{P} \sim 10^{19} \mathrm{GeV}$. One would hope that, like any other successful physical theory, a physically relevant quantum theory of gravity should lead to experimental predictions testable in the foreseeable future. In the QG case, however, such predictions may be both numerically suppressed and experimentally difficult to isolate. This is mainly due to the extremely weak nature of the gravitational interaction as compared with the rest of the known forces in nature. Specifically, the dimension-full coupling constant of gravity, the Newton constant $G_{N}=1 / M_{P}^{2}$, appears as a very strong suppression factor of any physical observable that could be associated with predictions of quantum gravity. If the feebleness of the gravitational interaction were to be combined with the exact conservation of all symmetries and properties in particle physics, one would arrive at the inescapable conclusion that a true "phenomenology of QG" should be considered wishful thinking.

In recent years, however, physicists have ventured into the idea that laws, such as Lorentz invariance and unitarity, which are characteristic of a flat space-time quantum field theory, may not be valid in a full quantum theory of curved space-time. For instance, the invariance of the laws of Nature under the CPT symmetry [1] (i.e. the combined action of charge conjugation $(\mathrm{C})$, parity-reflection $(\mathrm{P})$, and time-reversal $(\mathrm{T})$ ) may not be exact in the presence of QG effects. Indeed, the possibility of a violation of CPT invariance (CPTV) by QG has been raised in a number of theoretical models, that go beyond conventional local quantum field theoretic treatments of gravity [2, 3, 4, 5, 6, 7, 8].

CPT violating scenarios may be classified into two major categories, according to the specific way the symmetry is violated. The first category contains models in which CPTV is associated with (spontaneous) breaking of (linear) Lorentz symmetry [6] and/or locality of the interactions [7]. In these cases the generator of the CPT symmetry is a well-defined 
quantum mechanical operator, which however does not commute with the (effective) Hamiltonian of the system. In the second major category, the CPT operator, due to a variety of reasons, is ill-defined. The most notable example of such a type of CPTV is due to the entanglement of the matter theory with an "environment" of quantum gravitational degrees of freedom, leading to decoherence of matter [2, 4, 5, 8]. This decoherence, in turn, implies, by means of a powerful mathematical theorem due to Wald [9], that the quantum mechanical operator generating CPT transformations cannot be consistently defined. Wald refers to this phenomenon as "microscopic time irreversibility", which captures the essence of the effect. We shall refer to such a failure of CPT through decoherence as "intrinsic", in contradistinction to "extrinsic" violations, related to the non-commutativity of the matter Hamiltonian with a well-defined CPT generator [6, 7].

As emphasized recently in [8], this intrinsic CPTV leads to modifications to the concept of an "antiparticle". The resulting loss of particle-antiparticle identity in the neutral-meson system induces a breaking of the Einstein-Podolsky-Rosen (EPR) correlation imposed by Bose statistics. Specifically, in the CPT invariant case, the antiparticle of a given particle is defined as the state of equal mass (and life-time) and opposite values for all charges. If the CPT operator is well defined, such a state is obtained by the action of this operator on the corresponding particle state. If, however, the operator is ill-defined, the particle and antiparticle spaces should be thought of as independent subspaces of matter states. In such a case, the usual operating assumption that the electrically neutral meson states (Kaons or $B$ mesons) are indistinguishable from ("identical" to) their respective antiparticles $\left(\bar{K}^{0}\right.$ or $\left.\bar{B}^{0}\right)$ is relaxed. This, in turn, modifies the symmetry properties in the description of (neutral) meson entangled states, and may bring about deviations to their EPR correlations [8, 10]. We emphasize, though, that the associated intrinsic CPTV effects are treated as small perturbations, being attributed to quantum-gravitational physics. From now on, we will refer to this effect as the " $\omega$ effect", due to the complex parameter $\omega$ used in its parametrization.

It should be stressed at this point that the $\omega$ effect is distinct, both in its origin as well as the physical consequences, from analogous effects generated by the evolution of the system in the decoherence-inducing medium of QG, discussed in [5]. As already mentioned in [8], and will be discussed in detail in the next section, the $\omega$ effect appears immediately after the $\phi$ decay (at time $t=0$ ) due to the violation of the symmetry properties of the entangled Kaon state wave-function under permutations, assuming conservation of angular momentum. In 
other words, the $\omega$ effect provides an answer to the question: "What is the (symmetry of the) initial state of the $K^{0} \bar{K}^{0}$ system in the presence of a QG-decoherence inducing background ?" This is to be contrasted to the effects of [5] , stemming from the decoherent evolution of the system in the QG medium, which have been interpreted as a violation of angular momentum conservation. Our working hypothesis is that the QG medium conserves angular momentum, at least as far as the $\omega$ effect is concerned, extrapolating from no-hair theorems of classical macroscopic black holes.

In 8] it was assumed for simplicity that the intrinsic CPTV manifested itself only at the level of the wave-function describing the entangled two-meson state immediately after the decay of the initial resonance, whereas the subsequent time evolution of the system was taken to be due to an effective Hamiltonian. However, given that the origin of this intrinsic CPTV is usually attributed to the decoherent nature of QG, in the spirit discussed originally in [9], a complete description would necessitate the introduction of such "medium" effects in the time evolution as well. To accomplish such a description, at least for the purpose of providing a phenomenological framework for decoherent QG, no detailed knowledge of the underlying microscopic quantum gravity theory is necessary. This can be achieved following the so-called Lindblad or mathematical semi-groups approach to decoherence [11], which is a very efficient way of studying open systems in quantum mechanics [12]. The time irreversibility in the evolution of such semigroups, which is linked to decoherence, is inherent in the mathematical property of the lack of an inverse in the semigroup. This approach has been followed for the study of quantum-gravity decoherence in the case of neutral kaons [2, 4], as well as other probes, such as neutrinos [13, 14]. The Lindblad approach to decoherence does not require any detailed knowledge of the environment, apart from energy conservation, entropy increase and complete positivity of the (reduced) density matrix $\rho(t)$ of the subsystem under consideration. The basic evolution equation for the (reduced) density matrix of the subsystem in the Lindblad approach is linear in $\rho(t)$. We notice that the Lindblad part, expressing interaction with the "environment" cannot be written as a commutator (of a Hamiltonian function) with $\rho$. Environmental contributions that can be cast in Hamiltonian evolution (commutator form) are absorbed in an "effective" hamiltonian.

The purpose of this article is to extend the analysis of [8], where the intrinsic CPTV manifested itself only in the initial entangled meson state, to include QG environmental en- 
tanglement, by resorting to the above-mentioned Lindblad evolution formalism. Specifically, we shall compute various observables relevant for precision decoherence tests in a $\phi$-factory, placing emphasis on the possibility of disentangling the decoherent evolution parameters $\alpha, \beta$, and $\gamma$ of [2] from the $\omega$ parameter of [8], expressing the loss of particle-antiparticle identity mentioned above. The structure of the article is as follows: in section 2 we present our formalism, including notations and conventions, which agree with those used in [5]. In section 3 we describe the various observables of a $\phi$ factory that constitute sensitive probes of our $\omega$ and decoherence $\alpha, \beta, \gamma$ effects, and explain how the various effects (or bounds thereof) can be disentangled experimentally. In this section we restrict ourselves only to leading order corrections in the CPTV and decoherence effects, and ignore $\epsilon^{\prime}$ corrections. Such corrections do not affect the functional form of the CPTV and decoherent evolution terms of the various observables, and their inclusion is discussed in an Appendix. Conclusions and discussion are presented in section 4. Particular emphasis in the discussion is given in the contamination of the observable giving traditionally (i.e. ignoring decoherent CPTV evolution) the $\epsilon^{\prime}$ effects, by terms depending on the decoherence coefficients. Finally, as already mentioned, in an Appendix we give the complete formulae for the observables of section 3 , including $\epsilon^{\prime}$ corrections.

\section{FORMALISM}

In this section we shall follow the notation and conventions of [5, 15]. The CP violating parameters are defined as $\epsilon_{S}=\epsilon_{M}+\Delta, \epsilon_{L}=\epsilon_{M}-\Delta$, according to which the physical mass-eigenstates are expressed as

$$
\begin{aligned}
\left|K_{S}\right\rangle & =\frac{N_{S}}{\sqrt{2}}\left(\left(1+\epsilon_{S}\right)\left|K_{0}\right\rangle+\left(1-\epsilon_{S}\right)\left|\overline{K_{0}}\right\rangle\right) \\
\left|K_{L}\right\rangle & =\frac{N_{L}}{\sqrt{2}}\left(\left(1+\epsilon_{L}\right)\left|K_{0}\right\rangle-\left(1-\epsilon_{L}\right)\left|\overline{K_{0}}\right\rangle\right)
\end{aligned}
$$

where $N_{S, L}$ are positive normalization constants, $\epsilon_{M}$ is odd under CP, but even under CPT, and $\Delta$ is odd under both $\mathrm{CP}$ and CPT. Furthermore,

$$
\begin{aligned}
\epsilon_{i}^{ \pm} & =\epsilon_{i} \pm \frac{\beta}{d} \\
\epsilon_{i}^{ \pm *} & =\epsilon_{i}^{*} \pm \frac{\beta}{d^{*}}, \quad i=L, S
\end{aligned}
$$


with

$$
\begin{aligned}
d & =\Delta m+\frac{i}{2} \Delta \Gamma=|d| e^{i\left(\pi / 2-\phi_{S W}\right)} \\
& =((3.483 \pm 0.006)+i(3.668 \pm 0.003)) \times 10^{-15} \mathrm{GeV}
\end{aligned}
$$

and the super-weak angle [16] $\phi_{S W}=(43.5 \pm 0.7)^{\circ}$.

To find the correct expressions for the observables in the density matrix formalism of 2, 4, 5], matching the standard phenomenology in the CPT-conserving quantum mechanical case, we use the following parametrization for the amplitudes of the decay of $\left|K_{1}\right\rangle,\left|K_{2}\right\rangle \rightarrow$ $\left|\pi^{+} \pi^{-}\right\rangle[5]$ :

$$
\begin{aligned}
& \mathcal{M}\left(K_{1} \rightarrow \pi^{+} \pi^{-}\right)=\sqrt{2} A_{0} e^{i \delta_{0}}+\operatorname{Re} A_{2} e^{i \delta_{2}} \\
& \mathcal{M}\left(K_{2} \rightarrow \pi^{+} \pi^{-}\right)=i \operatorname{Im} A_{2} * e^{i \delta_{2}}
\end{aligned}
$$

where $A_{0}$ is real, and $\epsilon^{\prime}=\frac{i}{\sqrt{2}} \frac{\operatorname{Im} A_{2}}{A_{0}} e^{i \delta}, \delta \equiv \delta_{2}-\delta_{0}$, and $\left|\eta_{+-}\right|^{2} \equiv\left|\frac{\mathcal{M}\left(K_{L} \rightarrow \pi^{+} \pi^{-}\right)}{\mathcal{M}\left(K_{S} \rightarrow \pi^{+} \pi^{-}\right)}\right|^{2}=$ $\left|\epsilon_{L}+\frac{1}{\sqrt{2}} \frac{i \operatorname{Im} A_{2}}{A_{0}} e^{i \delta}\right|^{2}, \quad\left|\eta_{00}\right|^{2} \equiv\left|\frac{\mathcal{M}\left(K_{L} \rightarrow \pi^{0} \pi^{0}\right)}{\mathcal{M}\left(K_{S} \rightarrow \pi^{0} \pi^{0}\right)}\right|^{2}=\left|\epsilon_{L}-\sqrt{2} \frac{i \operatorname{Im} A_{2}}{A_{0}} e^{i \delta}\right|^{2}$. We also introduce the standard parametrization $\eta_{+-}=\left\langle\pi^{+} \pi^{-} \mid K_{L}\right\rangle /\left\langle\pi^{+} \pi^{-} \mid K_{S}\right\rangle=\left|\eta_{+-}\right| e^{i \phi_{+-}}$, where [16] $\left|\eta_{+-}\right|=(2.288 \pm 0.014) \times 10^{-3}, \phi_{+-}=(43.4 \pm 0.7)^{\circ}$. Similar parametrizations can be used for the corresponding rations representing Kaon decays to two neutral as well as three pions. The corresponding phases are denoted $\phi_{00}$ and $\phi_{3 \pi}$. We remind the reader that within quantum mechanics $\eta_{+-}=\epsilon_{L}+\epsilon^{\prime}, \eta_{00}=\epsilon_{L}-2 \epsilon^{\prime}$.

All the above numbers are extracted without assuming CPT invariance. In the presence of CPTV decoherence parameters $(\beta)$ this relation is modified, given that now $\epsilon_{L, S}$ are replaced by $\epsilon_{L, S}^{ \pm}(2.2)$. In that case [5], $\epsilon_{L}^{-}$is related to $\bar{\eta}_{+-}$, which replaces the quantum-mechanical $\eta_{+-}$defined above, through $\left|\bar{\eta}_{+-}\right| e^{i \phi_{+-}}=\epsilon_{L}^{-}+Y_{+-}$, where $Y_{+-}$includes the $\epsilon^{\prime}$ effects, $Y_{+-}=\left\langle\pi^{+} \pi^{-} \mid K_{2}\right\rangle /\left\langle\pi^{+} \pi^{-} \mid K_{1}\right\rangle$, with $K_{1}=\frac{1}{\sqrt{2}}\left(\left|K^{0}\right\rangle+\left|\bar{K}^{0}\right\rangle\right)$ and $K_{2}=\frac{1}{\sqrt{2}}\left(\left|K^{0}\right\rangle-\left|\bar{K}^{0}\right\rangle\right)$ the standard CP eigenstates. We will also use the abbreviations $\Delta \epsilon_{S L}=\epsilon_{S}-\epsilon_{L}$ and $\sum \epsilon_{S L}=\epsilon_{S}+\epsilon_{L}$, and $\bar{\Gamma}=\frac{1}{2}\left(\Gamma_{L}+\Gamma_{S}\right)$, as well as the definition [5]

$$
R_{L}=\left|\epsilon_{L}^{-}\right|^{2}+\frac{\gamma}{\Delta \Gamma}+4 \frac{\beta}{\Delta \Gamma} \operatorname{Im}\left(\frac{\epsilon_{L}^{-} d}{d^{*}}\right),
$$

which we shall make use of in the present article. We also note the current experimental values $\sqrt{R_{L}}=(2.30 \pm 0.035) \times 10^{-3}, 2 \operatorname{Re} \epsilon_{L}^{+}=(3.27 \pm 0.12) \times 10^{-3}$. Finally, the relevant density matrices read $\rho_{L}=\left|K_{L}\right\rangle\left\langle K_{L}\left|, \rho_{S}=\right| K_{S}\right\rangle\left\langle K_{S}\left|, \rho_{I}=\right| K_{S}\right\rangle\left\langle K_{L}\left|, \rho_{\bar{I}}=\right| K_{L}\right\rangle\left\langle K_{S}\right|$. In 
the present article we shall use values for the decoherence parameters $\alpha, \beta$, and $\gamma$, which saturate the experimental bounds obtained by the CPLEAR experiment [17]:

$$
\alpha<4.0 \times 10^{-17} \mathrm{GeV}, \quad|\beta|<2.3 \times 10^{-19} \mathrm{GeV}, \quad \gamma<3.7 \times 10^{-21} \mathrm{GeV}
$$

We note at this stage that in certain models [18], where complete positivity of the density matrix of the entangled kaon state has been invoked, there is only one non-vanishing decoherence parameter $(\alpha=\gamma)$ in the formalism of [2], which we adopt here. However, as the analysis of [18] has demonstrated, in such completely positive entangled models there are other parametrizations, with more decoherence parameters. Our intrinsic CPT-violation $\omega$-effects 8] can be easily incorporated in those formalisms, which however we shall not follow here. For our purposes, complete positivity is achieved as the special case of only one decoherence parameter $(\gamma=\alpha)$ in the formulae that follow. We emphasize, however, that the issue of complete positivity is not entirely clear in a quantum-gravity context, where the relevant master equation may even be non-linear [19].

In conventional formulations of entangled meson states [20, 21, 22] one imposes the requirement of Bose statistics for the state $K^{0} \bar{K}^{0}$ (or $B^{0} \bar{B}^{0}$ ), which implies that the physical neutral meson-antimeson state must be symmetric under the combined operation $C \mathcal{P}$, with $C$ the charge conjugation and $\mathcal{P}$ the operator that permutes the spatial coordinates. Specifically, assuming conservation of angular momentum, and a proper existence of the antiparticle state (denoted by a bar), one observes that, for $K^{0} \bar{K}^{0}$ states which are $C$-conjugates with $C=(-1)^{\ell}$ (with $\ell$ the angular momentum quantum number), the system has to be an eigenstate of $\mathcal{P}$ with eigenvalue $(-1)^{\ell}$. Hence, for $\ell=1$, we have that $C=-$, implying $\mathcal{P}=-$. As a consequence of Bose statistics this ensures that for $\ell=1$ the state of two identical bosons is forbidden [20]. However, these assumptions may not be valid if CPT symmetry is intrinsically violated, in the sense of the loss of particle-antiparticle identity. In such a case $\bar{K}^{0}$ cannot be considered as identical to $K^{0}$, and thus the requirement of $C \mathcal{P}=+$, imposed by Bose-statistics, is relaxed. Therefore, the initial state after the $\phi$ decay, when expressed in terms of mass-eigenstates contains, in addition to the standard $K_{L} K_{S}$ terms, otherwise forbidden terms of the type $K_{L} K_{L}, K_{S} K_{S}$ :

$$
\begin{aligned}
|i\rangle & =C\left[\left(\left|K_{S}(\vec{k}), K_{L}(-\vec{k})\right\rangle-\left|K_{L}(\vec{k}), K_{S}(-\vec{k})\right\rangle\right)\right. \\
& \left.+\omega\left(\left|K_{S}(\vec{k}), K_{S}(-\vec{k})\right\rangle-\left|K_{L}(\vec{k}), K_{L}(-\vec{k})\right\rangle\right)\right]
\end{aligned}
$$


where $\mathrm{C}$ is to be computed, and $\omega$ is a complex parameter. We hasten to emphasize at this stage that the genuine, quantum gravity induced $\omega$-effect, due to the loss of particleantiparticle identity, should not be confused with the ordinary C-even-background effects that have been studied in [18]. In fact the $\omega$-effect can be easily disentangled from background effects, as has been discussed in [8].

Under the non-unitary decoherent Lindblad evolution [11], appropriately tailor to the present problem [4, 5]

$$
\partial_{t} \rho(t)=i \rho(t) H-i H^{\dagger} \rho(t)+\widehat{\delta H} \rho(t)
$$

where $H$ denotes the (non-hermitian) Hamiltonian of the system (taking decay into account) and $\widehat{\delta H}$ contains the decoherent effects, the initial state evolves to a mixed state, which assumes the general structure [5]

$$
\rho=\sum_{i, j} A_{i j} \rho_{i} \otimes \rho_{j}, \quad i, j=S, L
$$

where the constants $A_{i j}$ depend on the decoherence parameters.

The relevant observables are computed by means of double-decay rates with the following generic structure

$$
\mathcal{P}\left(f_{1}, \tau_{1} ; f_{2}, \tau_{2}\right)=\sum_{i j} A_{i j} \operatorname{tr}\left[\rho_{i} \mathcal{O}_{f_{1}}\right] \operatorname{tr}\left[\rho_{j} \mathcal{O}_{f_{2}}\right] e^{-\lambda_{i} \tau_{1}-\lambda_{j} \tau_{2}}
$$

where the constants $\lambda_{i}, \lambda_{j}$ also depend on the decoherence parameters.

The double decay rate $\mathcal{P}\left(f_{1}, \tau ; f_{2}, \tau\right)$ interpolated at equal times $\tau_{1}=\tau_{2}=\tau$ is a quantity that pronounces the unusual time dependences of the decoherent evolution, which will also be calculated in this work.

Finally, of particular experimental interest are also integrated distributions at fixed time intervals $\Delta \tau=\tau_{2}-\tau_{1}>0$ (which we can assume for our purposes here):

$$
\overline{\mathcal{P}}\left(f_{1} ; f_{2} ; \Delta \tau>0\right)=\frac{1}{2} \int_{\Delta \tau}^{\infty} d\left(\tau_{1}+\tau_{2}\right) \mathcal{P}\left(f_{1}, \tau_{1} ; f_{2}, \tau_{2}\right)
$$

where the factor $\frac{1}{2}$ originates from the Jacobian when changing variables $\left(\tau_{1}, \tau_{2}\right) \rightarrow\left(\tau_{1}+\right.$ $\left.\tau_{2}, \Delta \tau\right)$.

In the particular case of a $\phi$ factory that we analyze here, the pertinent density matrix describing the decay of one kaon of momentum $\vec{k}$ at time $\tau_{1}$ to a final state $f_{1}$ and the other 
of momentum $-\vec{k}$ at time $\tau_{2}$ to a final state $f_{2}$ is given by

$$
\begin{aligned}
\rho= & \rho_{S} \otimes \rho_{L}\left(1+\omega \Delta \epsilon_{S L}+\omega^{*} \Delta \epsilon_{S L}^{*}\right) e^{-\Gamma_{L} \tau_{2}-\Gamma_{S} \tau_{1}}+\rho_{L} \otimes \rho_{S}\left(1-\omega \Delta \epsilon_{S L}-\omega^{*} \Delta \epsilon_{S L}^{*}\right) e^{-\Gamma_{L} \tau_{1}-\Gamma_{S} \tau_{2}} \\
& -\left(\rho_{I} \otimes \rho_{\bar{I}}\left(1+\omega \Delta \epsilon_{S L}-\omega^{*} \sum \epsilon_{S L}^{*}\right) e^{-i \Delta m\left(\tau_{1}-\tau_{2}\right)}\right. \\
& \left.+\rho_{\bar{I}} \otimes \rho_{I}\left(1-\omega \Delta \epsilon_{S L}+\omega^{*} \sum \epsilon_{S L}^{*}\right) e^{+i \Delta m\left(\tau_{1}-\tau_{2}\right)}\right) e^{-(\bar{\Gamma}+\alpha-\gamma)\left(\tau_{1}+\tau_{2}\right)} \\
& -\left(\left(|\omega|^{2}-\frac{i \alpha}{\Delta m}\right) \rho_{I} \otimes \rho_{I} e^{-i \Delta m\left(\tau_{1}+\tau_{2}\right)}+\left(|\omega|^{2}+\frac{i \alpha}{\Delta m}\right) \rho_{\bar{I}} \otimes \rho_{\bar{I}} e^{+i \Delta m\left(\tau_{1}+\tau_{2}\right)}\right) e^{-(\bar{\Gamma}+\alpha-\gamma)\left(\tau_{1}+\tau_{2}\right)} \\
& +\left(|\omega|^{2}-\frac{2 \gamma}{\Delta \Gamma}\right) \rho_{S} \otimes \rho_{S} e^{-\Gamma_{S}\left(\tau_{1}+\tau_{2}\right)}+\left(|\omega|^{2}+\frac{2 \gamma}{\Delta \Gamma}\right) \rho_{L} \otimes \rho_{L} e^{-\Gamma_{L}\left(\tau_{1}+\tau_{2}\right)} \\
& +\rho_{I} \otimes \rho_{S} e^{-i \Delta m \tau_{1}-\Gamma_{S} \tau_{2}-(\bar{\Gamma}+\alpha-\gamma) \tau_{1}}\left(\omega-\frac{2 \beta}{d}\right)-\rho_{S} \otimes \rho_{I} e^{-i \Delta m \tau_{2}-\Gamma_{S} \tau_{1}-(\bar{\Gamma}+\alpha-\gamma) \tau_{2}}\left(\omega+\frac{2 \beta}{d}\right) \\
& +\rho_{\bar{I}} \otimes \rho_{S} e^{+i \Delta m \tau_{1}-\Gamma_{S} \tau_{2}-(\bar{\Gamma}+\alpha-\gamma) \tau_{1}}\left(\omega^{*}-\frac{2 \beta}{d^{*}}\right)-\rho_{S} \otimes \rho_{\bar{I}} e^{+i \Delta m \tau_{2}-\Gamma_{S} \tau_{1}-(\bar{\Gamma}+\alpha-\gamma) \tau_{2}}\left(\omega^{*}+\frac{2 \beta}{d^{*}}\right) \\
& +\rho_{I} \otimes \rho_{L} e^{-i \Delta m \tau_{1}-\Gamma_{L} \tau_{2}-(\bar{\Gamma}+\alpha-\gamma) \tau_{1}}\left(\omega^{*}+\frac{2 \beta}{d^{*}}\right)-\rho_{L} \otimes \rho_{I} e^{-i \Delta m \tau_{2}-\Gamma_{L} \tau_{1}-(\bar{\Gamma}+\alpha-\gamma) \tau_{2}}\left(\omega^{*}-\frac{2 \beta}{d^{*}}\right) \\
& +\rho_{\bar{I}} \otimes \rho_{L} e^{+i \Delta m \tau_{1}-\Gamma_{L} \tau_{2}-(\bar{\Gamma}+\alpha-\gamma) \tau_{1}}\left(\omega+\frac{2 \beta}{d}\right)-\rho_{L} \otimes \rho_{\bar{I}} e^{+i \Delta m \tau_{2}-\Gamma_{L} \tau_{1}-(\bar{\Gamma}+\alpha-\gamma) \tau_{2}}\left(\omega-\frac{2 \beta}{d}\right)
\end{aligned}
$$

from the which the expression for the various constants in Eq.(2.10) may be gleaned. An important remark is now in order. Notice that above we have treated $|\omega|^{2}$ effects as being of comparable order to decoherence $\alpha, \gamma$, and have kept only terms at most linear in the decoherence parameters. This is a very simplifying assumption, which, due to a lack of a microscopic theory of QG, cannot be made rigorous. However, as we shall discuss below, a reasonable a posteriori justification of this approximation may come from the fact that such terms appear on an equal footing as medium-generated corrections to certain physically important terms of (2.12) to be analyzed below.

In Eq.(2.12) we notice that, starting from the third line, terms of entirely novel type make their appearance. Such terms are due to both the intrinsic CPTV loss of particle-antiparticle identity ( $\omega$-related) and decoherent evolution $(\alpha, \beta, \gamma,-$ related). Such terms persist in the limit $\tau_{1}=\tau_{2}=0$, due to a specific choice of boundary conditions in the solution of the appropriate density-matrix evolution equation (2.8), expressing the omnipresence of the QG space-time foam effects. In particular, terms linear in $\omega$ are always accompanied by the parameter $\beta$, whereas terms quadratic in $\omega$ always combine with the $\alpha$ or $\gamma$ parameters. Of particular physical importance are the otherwise forbidden terms $\rho_{L} \otimes \rho_{L}$ and $\rho_{S} \otimes \rho_{S}$. 
At first sight, it is tempting to interpret [5] such terms as signaling a subtle breakdown of angular momentum conservation, in addition to the CPTV introduced by the "medium". That seems to be an inescapable conclusion, if one were to begin the time evolution from a purely antisymmetric wave function (i.e. with $\omega=0$ in (2.7)), as assumed in [5], which is an eigenstate of the orbital angular momentum $L$ with eigenvalue $\ell=1$. Since, then, the medium generates in the final state forbidden terms violating this last property, one interprets this as non-conservation of $L$. However, if one accepts [8] that the intrinsic CPTV affects not only the evolution but also the concept of the antiparticle, such terms are present already in the initial state. In such a case, there is no issue of non-conservation of angular momentum; the only effect of the time evolution is to simply modify the relative weights between $\rho_{L} \otimes \rho_{L}$ and $\rho_{S} \otimes \rho_{S}$ terms.

One can therefore say that QG may behave as a CPTV medium differentiating between particles and antiparticles. From a physical point of view one may even draw a vague analogy between QG media and "regenerators", in the following sense: exactly as the regenerator differentiates between $K^{0}$ and $\bar{K}^{0}$, by means of different relative interactions, in a similar way QG acts differently on particles and antiparticles; however, unlike the regenerator case, where the experimentalist can control its position, due to their universal nature, QG effects are present even in the initial state after the $\phi$ decay.

\section{OBSERVABLES AT $\phi$ FACTORIES}

In this section we present a detailed study of a variety of observables measurable at $\phi$ factories, and determine their dependence on both intrinsic $\operatorname{CPTV}(\omega)$ and decoherence $(\alpha$, $\beta, \gamma)$ parameters, in an attempt to disentangle and separately constrain the two possible effects. Specifically, we will derive expressions for the double-decay rates of Eq.(2.10) and their integrated counter-parts of Eq.(2.11), for the cases of identical as well as general (nonidentical) final states. We will restrict ourselves to linear effects in $\alpha, \beta$, $\gamma$, while keeping terms linear and quadratic in $\omega$, for the reasons explained in the previous section. For our purposes here we shall be interested in the decays of $K_{L, S}$ to final states consisting of two pions, $\pi^{+} \pi^{-}$or $\pi^{0}$, three pions, as well as semileptonic decays to $\ell^{+} \pi^{-} \nu$ or $\ell^{-} \pi^{+} \bar{\nu}$. In this section we shall also ignore explicit $\epsilon^{\prime}$ effects. Such effects appear in the branching ratios $\eta$ of various pion channels, and their inclusion affects the form of the associated observables. 
We present complete formulae for the relevant double-decay time distributions, including such effects, in an Appendix. As we note there, the inclusion of such effects does not affect significantly the functional form of the decoherent/CPTV evolution.

In the density-matrix formalism of [2, 5] the relevant observables are given by

$$
\begin{aligned}
& \mathcal{O}_{+-}=\left(\begin{array}{cr}
\mathcal{X}_{+-} & \mathcal{Y}_{+-} \\
\mathcal{Y}_{+-}^{*} & \left|\operatorname{Im} A_{2} e^{i \delta}\right|^{2}
\end{array}\right) \\
& \mathcal{O}_{00}=\left(\begin{array}{ll}
\mathcal{X}_{00} & \mathcal{Y}_{00} \\
\mathcal{Y}_{00}^{*} & \left|-2 i \operatorname{Im} A_{2} e^{i \delta}\right|^{2}
\end{array}\right) \\
& \mathcal{O}_{\ell^{+}}=\frac{|a|^{2}}{2}\left(\begin{array}{rr}
1 & 1 \\
1 & 1
\end{array}\right) \\
& \mathcal{O}_{\ell^{-}}=\frac{|a|^{2}}{2}\left(\begin{array}{rr}
1 & -1 \\
-1 & 1
\end{array}\right) \\
& \mathcal{O}_{3 \pi}=\left|X_{3 \pi}\right|^{2}\left(\begin{array}{ll}
0 & 0 \\
0 & 1
\end{array}\right) .
\end{aligned}
$$

where $\mathcal{X}_{+-}=\left|\sqrt{2} A_{0}+\operatorname{Re} A_{2} e^{i \delta}\right|^{2}, \mathcal{Y}_{+-}=\left(\sqrt{2} A_{0}+\operatorname{Re} A_{2} e^{-i \delta}\right)\left(i \operatorname{Im} A_{2} e^{i \delta}\right), \mathcal{X}_{00}=\mid \sqrt{2} A_{0}-$ $\left.2 \operatorname{Re} A_{2} e^{i \delta}\right|^{2}$ and $\mathcal{Y}_{00}=\left(\sqrt{2} A_{0}-2 \operatorname{Re} A_{2} e^{-i \delta}\right)\left(-2 i \operatorname{Im} A_{2} e^{i \delta}\right)$, and $X_{3 \pi}=\left\langle 3 \pi \mid K_{2}\right\rangle$, in an obvious shorthand notation $O_{f}$, where $f$ denotes the observable associated with a Kaon decay leading to $f$ final state, for instance $f=+-$ denotes decay to $\pi^{+} \pi^{-}, f=00$ denotes decay to two $\pi^{0}, f=\ell^{+}$denotes semileptonic decay $\ell^{+} \pi^{-} \nu$ etc. Notice that if $\epsilon^{\prime}$ effects are ignored, which we shall do here for brevity, then we do not distinguish between the observables for the two cases of the two-pion final states.

Note that above we ignored CPT violating effects in the decay amplitudes to the final states. If such effects are included then the observables acquire the form [5]:

$$
\begin{aligned}
& \mathcal{O}_{+-}=\left|X_{+-}\right|^{2}\left(\begin{array}{cc}
1 & Y_{+-} \\
Y_{+-}^{*} & \left|Y_{+-}\right|^{2}
\end{array}\right) \\
& \mathcal{O}_{00}=\left|X_{00}\right|^{2}\left(\begin{array}{cc}
1 & Y_{00} \\
Y_{00}^{*} & \left|Y_{00}\right|^{2}
\end{array}\right) \\
& \mathcal{O}_{\ell^{+}}=\frac{|a+b|^{2}}{2}\left(\begin{array}{cc}
1 & 1 \\
1 & 1
\end{array}\right),
\end{aligned}
$$




$$
\begin{aligned}
& \mathcal{O}_{\ell^{-}}=\frac{|a-b|^{2}}{2}\left(\begin{array}{cc}
1 & -1 \\
-1 & 1
\end{array}\right), \\
& \mathcal{O}_{3 \pi}=\left|X_{3 \pi}\right|^{2}\left(\begin{array}{cc}
\left|Y_{3 \pi}\right|^{2} & Y_{3 \pi}^{*} \\
Y_{3 \pi} & 1
\end{array}\right) .
\end{aligned}
$$

where $X=<\pi \pi \mid K_{+}>, Y=\frac{<\pi \pi \mid K_{-}>}{<\pi \pi \mid K_{+}>}$, and more specifically $Y_{+-}=\left(\frac{\operatorname{Re}\left(B_{0}\right)}{A_{0}}\right)+\epsilon^{\prime}, Y_{+-}=$ $\left(\frac{\operatorname{Re} B_{0}}{A_{0}}\right)-2 \epsilon^{\prime}, Y_{3 \pi}=\frac{\left\langle 3 \pi \mid K_{1}\right\rangle}{\left\langle 3 \pi \mid K_{2}\right\rangle}$, and $\operatorname{Re}(a / b)$ and $\operatorname{Re}\left(B_{0}\right) / A_{0}$ parametrize CPT violation in the decay amplitudes to the appropriate final states.

\section{A. Identical final states}

Consider first the case where the kaons decay to $\pi^{+} \pi^{-}$. Ignoring CPTV in the decays and $\epsilon^{\prime}$ effects, the relevant observable, $\mathcal{O}_{+-}$, is obtained from the first of Eq.(3.1) by setting $A_{2}=0$, namely

$$
\mathcal{O}_{+-}=\left(\begin{array}{cc}
2 A_{0}^{2} & 0 \\
0 & 0
\end{array}\right)
$$

The corresponding double-decay rate is then

$$
\begin{aligned}
\mathcal{P} & \left(\pi^{+} \pi^{-}, \tau_{1} ; \pi^{+} \pi^{-}, \tau_{2}\right)=2 A_{0}^{4}\left[R_{L}\left(e^{-\Gamma_{L} \tau_{2}-\Gamma_{S} \tau_{1}}+e^{-\Gamma_{L} \tau_{1}-\Gamma_{S} \tau_{2}}\right)\right. \\
& -2\left|\bar{\eta}_{+-}\right|^{2} \cos \left(\Delta m\left(\tau_{1}-\tau_{2}\right)\right) e^{-(\bar{\Gamma}+\alpha-\gamma)\left(\tau_{1}+\tau_{2}\right)}+\left(|\omega|^{2}-\frac{2 \gamma}{\Delta \Gamma}-\frac{4 \beta}{|d|}\left|\bar{\eta}_{+-}\right| \frac{\sin \phi_{+-}}{\cos \phi_{S W}}\right) e^{-\Gamma_{S}\left(\tau_{1}+\tau_{2}\right)} \\
& +\frac{4 \beta\left|\bar{\eta}_{+-}\right|}{|d|} \sin \left(\Delta m \tau_{1}+\phi_{+-}-\phi_{S W}\right) e^{-(\bar{\Gamma}+\alpha-\gamma) \tau_{1}-\Gamma_{S} \tau_{2}} \\
& +\frac{4 \beta\left|\bar{\eta}_{+-}\right|}{|d|} \sin \left(\Delta m \tau_{2}+\phi_{+-}-\phi_{S W}\right) e^{-(\bar{\Gamma}+\alpha-\gamma) \tau_{2}-\Gamma_{S} \tau_{1}} \\
& \left.+2|\omega|\left|\bar{\eta}_{+-}\right|\left(\cos \left(\tau_{1}+\phi_{+-}-2 \Omega\right) e^{-\Gamma_{S}\left(\tau_{2}\right)-(\bar{\Gamma}+\alpha-\gamma) \tau_{1}}-\cos \left(\tau_{2}+\phi_{+-}-\Omega\right) e^{-\Gamma_{S} \tau_{1}-(\bar{\Gamma}+\alpha-\gamma) \tau_{2}}\right)\right]
\end{aligned}
$$

The integrated observable $\overline{\mathcal{P}}\left(f_{1}, f_{2}, \Delta \tau\right)$ is obtained by integrating over $\left(\tau_{1}+\tau_{2}\right)$ keeping $\Delta \tau$ fixed. The result is:

$$
\begin{aligned}
& \overline{\mathcal{P}}\left(\pi^{+} \pi^{-} ; \pi^{+} \pi^{-} ; \Delta \tau\right)=A_{0}^{4}\left[R_{L} \frac{e^{-\Delta \tau \Gamma_{L}}+e^{-\Delta \tau \Gamma_{S}}}{\Gamma_{L}+\Gamma_{S}}\right. \\
& -\left|\bar{\eta}_{+-}\right|^{2} \cos (\Delta m \Delta \tau) \frac{e^{-(\bar{\Gamma}+\alpha-\gamma) \Delta \tau}}{(\bar{\Gamma}+\alpha-\gamma)}+\left(|\omega|^{2}-\frac{2 \gamma}{\Delta \Gamma}-\frac{4 \beta}{|d|}\left|\bar{\eta}_{+-}\right| \frac{\sin \phi_{+-}}{\cos \phi_{S W}}\right) \frac{e^{-\Gamma_{S} \Delta \tau}}{2 \Gamma_{S}}
\end{aligned}
$$




$$
\begin{aligned}
& +\frac{1}{\Delta m^{2}+(\bar{\Gamma}+\alpha-\gamma)^{2}+2(\bar{\Gamma}+\alpha-\gamma) \Gamma_{S}+\Gamma_{S}^{2}} \\
& \times\left[\frac { 4 \beta | \overline { \eta } _ { + - } | } { | d | } \left(\left(\Delta m \cos \left(\phi_{+-}-\phi_{S W}\right)+\left(\bar{\Gamma}+\alpha-\gamma+\Gamma_{S}\right) \sin \left(\phi_{+-}-\phi_{S W}\right)\right) e^{-\Delta \tau \Gamma_{S}}\right.\right. \\
& +e^{-\Delta \tau(\bar{\Gamma}+\alpha-\gamma)}\left(\Delta m \cos \left(\Delta m \Delta \tau+\phi_{+-}-\phi_{S W}\right)\right. \\
& \left.\left.+\left(\bar{\Gamma}+\alpha-\gamma+\Gamma_{S}\right) \sin \left(\Delta m \Delta \tau+\phi_{+-}-\phi_{S W}\right)\right)\right) \\
& +2|\omega|\left|\bar{\eta}_{+-}\right| e^{-\Delta \tau \Gamma_{S}}\left(\left(\bar{\Gamma}+\alpha-\gamma+\Gamma_{S}\right) \cos \left(\phi_{+-}-\Omega\right)-\Delta m \sin \left(\phi_{+-}-\Omega\right)\right) \\
& -2|\omega|\left|\bar{\eta}_{+-}\right| e^{(\bar{\Gamma}+\alpha-\gamma) \Delta \tau}\left(\left(\bar{\Gamma}+\alpha-\gamma+\Gamma_{S}\right) \cos \left(\Delta m \Delta \tau+\phi_{+-}-\Omega\right)\right. \\
& \left.\left.\left.-\Delta m \sin \left(\Delta m \Delta \tau+\phi_{+-}-\Omega\right)\right)\right]\right]
\end{aligned}
$$

which we plot in Figs. 1, 2. In the figures we assume rather large vales of $|\omega|$, of order $\left|\bar{\eta}_{+_{-}}\right|$. As evidenced by looking at the curves for relatively large values of $\Delta \tau$, this assumption, together with the assumed values of the decoherence parameters (2.6), allows for a rather clear disentanglement of the decoherence plus $\omega$ situation from that with only $\omega$ effects present (i.e. unitary evolution). Indeed, in the latter case, the result for the pertinent time-integrated double-decay rate quickly converges to the quantum-mechanical situation, in contrast to the decoherent case. Moreover, the non-zero value of the time-integrated asymmetries at $\Delta \tau=0$, is another clear deviation from the quantum mechanical case, which in the (unitary evolution) case with only $\omega \neq 0$ is pronounced, due to the presence of $\left|\bar{\eta}_{+-}\right|$factors [8]].

We notice at this stage, though, that this may not be the case in an actual quantum gravity foam situation. For instance, it has been argued [18] that if complete positivity is imposed for the density matrix of entangled states, then the only non-trivial decoherence parameter would be $\alpha=\gamma>0$, whilst $\beta=0$. It is not inconceivable that $|\omega|^{2}$ are of the same order as $2 \gamma / \Delta \Gamma$, in which case, for all practical purposes the integrated curve for the two-pion double decay rate (3.4) would pass through zero for $\Delta \tau=0$. In such a situation, one should also look at the linear in $\omega$ interference terms for $\Delta \tau \neq 0$, in order to disentangle the $\omega$ from $\gamma$ effects. Lacking a fundamental microscopic theory underlying these phenomenological considerations, however, which would make definite predictions on the relative order of the various CPTV effects, we are unable to make more definite statements at present.

We next look at the semileptonic decays of Kaons. First we consider the case where both Kaons decay to $\ell^{+}$. As discussed in the beginning of the section, (3.1) the relevant 

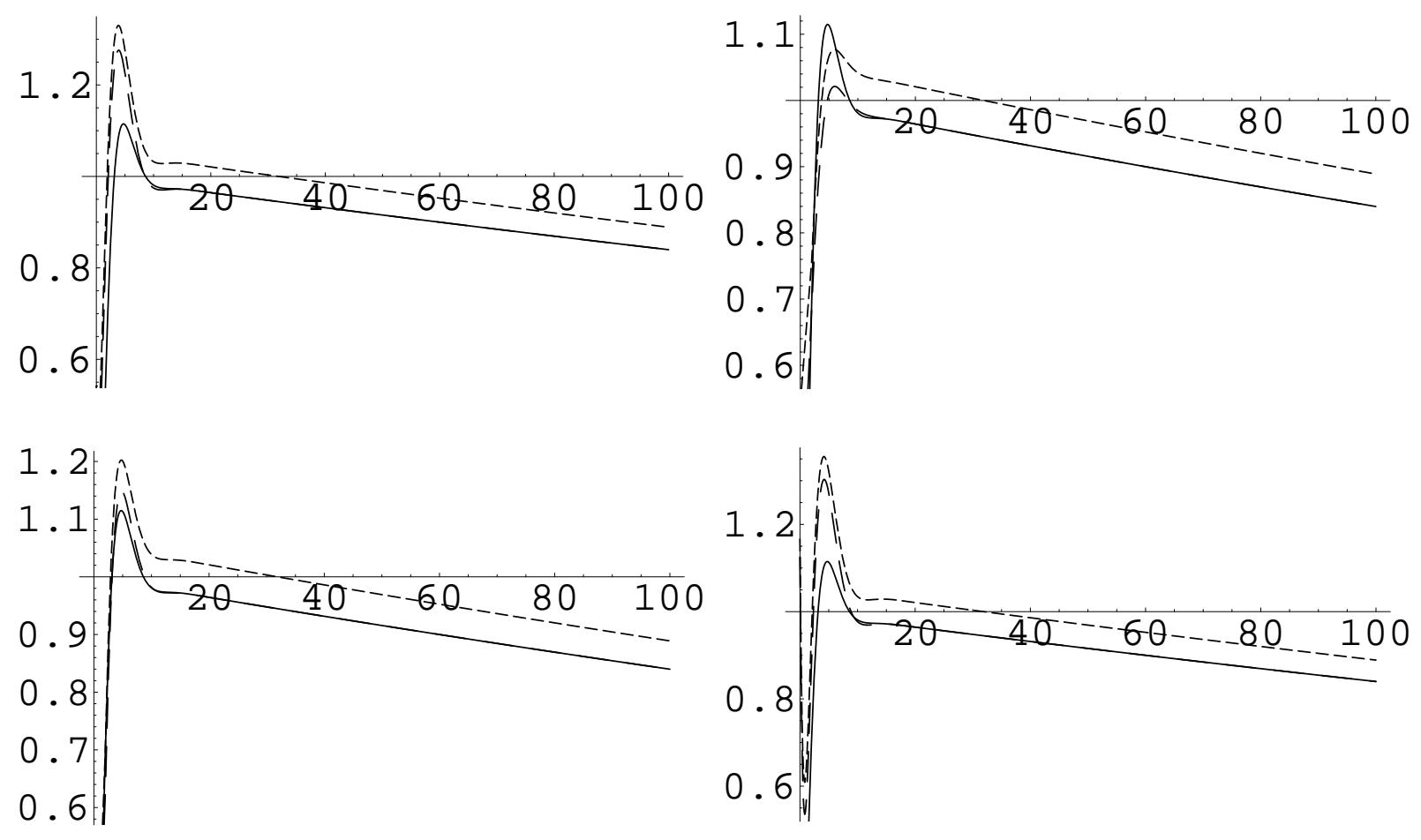

FIG. 1: Plots of $\overline{\mathcal{P}}\left(\pi^{ \pm}, \pi^{ \pm}, \Delta \tau\right)$ vs. $\Delta \tau$ extended to a large range of $\Delta \tau$. The dashed curves are with $\alpha, \beta, \gamma, \omega$ all non-zero, the long-dashed curve has only $\omega \neq 0$, whilst the solid curve represents $\alpha, \beta, \gamma, \omega=0$. Here and in all subsequent figures the values for $\alpha, \beta$, and $\gamma$ saturate the CPLEAR bound of Eq. (2.6). The values for $\omega$ and $\Omega$ are (from left to right, and top to bottom) i) $|\omega|=\left|\bar{\eta}_{+-}\right|$, $\Omega=\phi_{+-}-0.16 \pi$, ii) $|\omega|=\left|\bar{\eta}_{+-}\right|, \Omega=\phi_{+-}+0.95 \pi$, iii) $|\omega|=0.5\left|\bar{\eta}_{+-}\right|, \Omega=\phi_{+-}+0.16 \pi$, iv) $|\omega|=1.5\left|\bar{\eta}_{+-}\right|, \Omega=\phi_{+-} . \overline{\mathcal{P}}$ is in units of $\left|\bar{\eta}_{+-}\right|^{2} \tau_{s} 4 A_{0}^{4}$ and $\Delta \tau$ is in units of $\tau_{S}$.

observable is $\mathcal{O}_{l+}$,

$$
\mathcal{O}_{l+}=\frac{|a|^{2}}{2}\left(\begin{array}{ll}
1 & 1 \\
1 & 1
\end{array}\right)
$$

Recalling that $\epsilon_{L}=\epsilon_{M}-\Delta$ and $\epsilon_{S}=\epsilon_{M}+\Delta$ so $\sum\left(\epsilon_{S L}+\epsilon_{S L}^{*}\right)=4 \operatorname{Re} \epsilon_{M}$ we have for the double-decay rate:

$$
\begin{aligned}
\mathcal{P}\left(l^{ \pm}, \tau_{1} ; l^{ \pm}, \tau_{2}\right)= & \frac{a^{4}}{8}\left[\left(1 \pm 4 \operatorname{Re}\left(\epsilon_{M}\right)\right)\left(e^{-\Gamma_{L} \tau_{2}-\Gamma_{S} \tau_{1}}+e^{-\Gamma_{L} \tau_{1}-\Gamma_{S} \tau_{2}}\right)\right. \\
& -2\left(1 \pm 4 \operatorname{Re}\left(\epsilon_{M}\right)\right) \cos \left(\Delta m\left(\tau_{1}-\tau_{2}\right)\right) e^{-(\bar{\Gamma}+\alpha-\gamma)\left(\tau_{1}+\tau_{2}\right)} \\
& -2|\omega|^{2} \cos \left(\Delta m\left(\tau_{1}+\tau_{2}\right)\right) e^{-(\bar{\Gamma}+\alpha-\gamma)\left(\tau_{1}+\tau_{2}\right)}+|\omega|^{2}\left(e^{-\Gamma_{S}\left(\tau_{1}+\tau_{2}\right)}+e^{-\Gamma_{L}\left(\tau_{1}+\tau_{2}\right)}\right) \\
& +\frac{2 \gamma}{\Delta \Gamma}\left(e^{-\Gamma_{L}\left(\tau_{1}+\tau_{2}\right)}-e^{-\Gamma_{S}\left(\tau_{1}+\tau_{2}\right)}\right)+\frac{2 \alpha}{\Delta m} \sin \left(\Delta m\left(\tau_{1}+\tau_{2}\right)\right) e^{-(\bar{\Gamma}+\alpha-\gamma)\left(\tau_{1}+\tau_{2}\right)}
\end{aligned}
$$



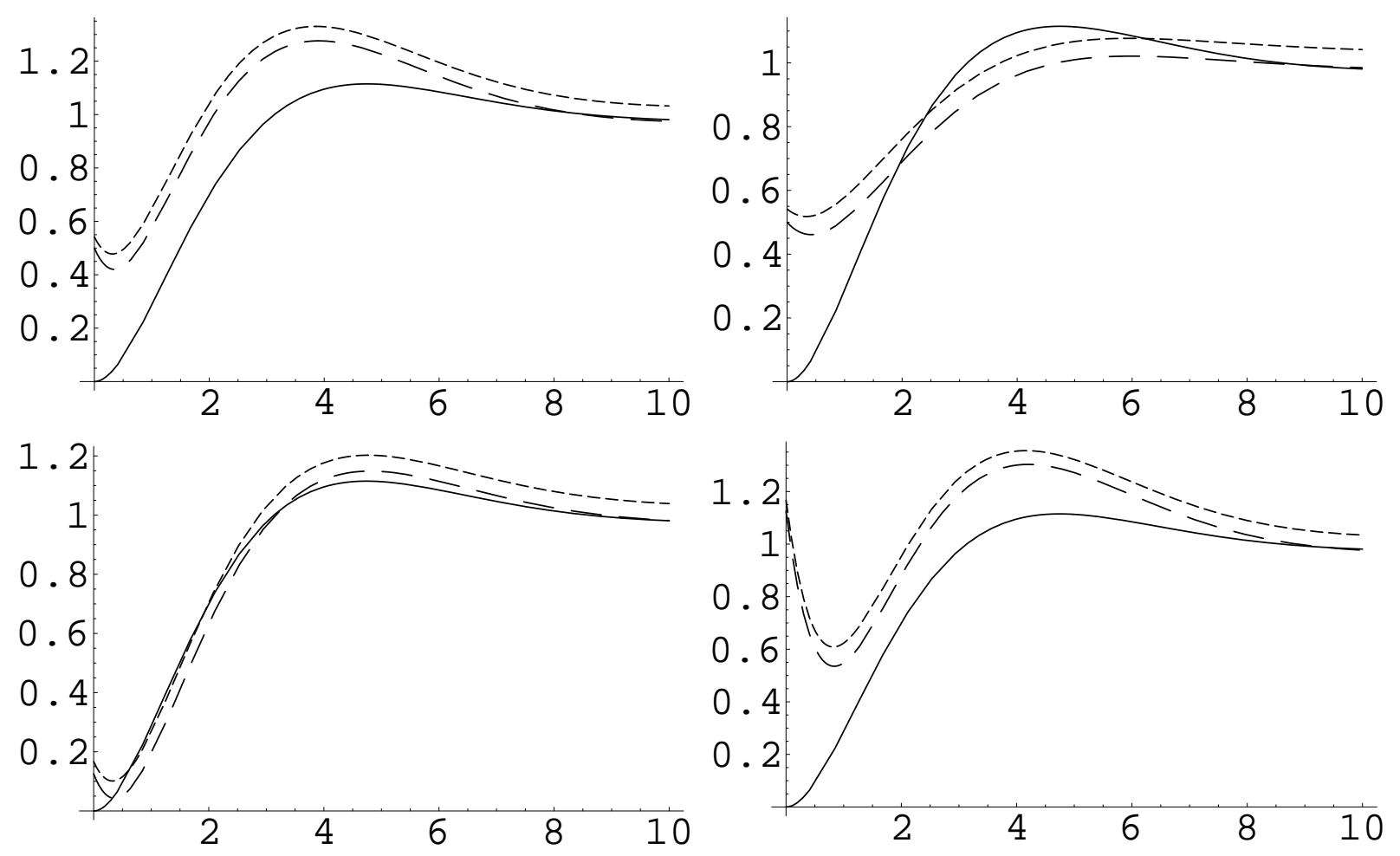

FIG. 2: As in Fig. 1, but shown in detail for small values of $\Delta \tau$ near the origin, to illustrate the difference between the various cases. Again, the dashed curves are with $\alpha, \beta, \gamma, \omega$ all non-zero, the long-dashed curve has only $\omega \neq 0$, whilst the solid curve represents $\alpha, \beta, \gamma, \omega=0$.

$$
\begin{aligned}
& \pm\left(\frac{4 \beta}{|d|} \sin \left(\Delta m \tau_{1}-\phi_{S W}\right)+2|\omega| \cos \left(\Delta m \tau_{1}-\Omega\right)\right) e^{-(\bar{\Gamma}+\alpha-\gamma) \tau_{1}} e^{-\Gamma_{S} \tau_{2}} \\
& \pm\left(\frac{4 \beta}{|d|} \sin \left(\Delta m \tau_{2}-\phi_{S W}\right)-2|\omega| \cos \left(\Delta m \tau_{2}-\Omega\right)\right) e^{-(\bar{\Gamma}+\alpha-\gamma) \tau_{2}} e^{-\Gamma_{S} \tau_{1}} \\
& \pm\left(\frac{4 \beta}{|d|} \sin \left(\Delta m \tau_{1}+\phi_{S W}\right)+2|\omega| \cos \left(\Delta m \tau_{1}+\Omega\right)\right) e^{-(\bar{\Gamma}+\alpha-\gamma) \tau_{1}} e^{-\Gamma_{L} \tau_{2}} \\
& \left. \pm\left(\frac{4 \beta}{|d|} \sin \left(\Delta m \tau_{2}+\phi_{S W}\right)-2|\omega| \cos \left(\Delta m \tau_{2}+\Omega\right)\right) e^{-(\bar{\Gamma}+\alpha-\gamma) \tau_{2}} e^{-\Gamma_{L} \tau_{1}}\right]
\end{aligned}
$$

and the integrated quantity reads:

$$
\begin{gathered}
\overline{\mathcal{P}}\left(l^{ \pm} ; l^{ \pm}, \Delta \tau\right)=\frac{a^{4}}{16}\left[2\left(1 \pm 4 \operatorname{Re} \epsilon_{M}\right) \frac{e^{-\Gamma_{L} \Delta \tau}+e^{-\Gamma_{S} \Delta \tau}}{\Gamma_{S}+\Gamma_{L}}\right. \\
-\left(1 \pm 4 \operatorname{Re} \epsilon_{M}\right) 2 \cos (\Delta m \Delta \tau) \frac{e^{-(\bar{\Gamma}+\alpha-\gamma) \Delta \tau}}{(\bar{\Gamma}+\alpha-\gamma)} \\
-2|\omega|^{2} e^{-\Delta \tau(\bar{\Gamma}+\alpha-\gamma)} \frac{(\bar{\Gamma}+\alpha-\gamma) \cos (\Delta m \Delta \tau)-\Delta m \sin (\Delta m \Delta \tau)}{(\bar{\Gamma}+\alpha-\gamma)^{2}+\Delta m^{2}}
\end{gathered}
$$




$$
\begin{aligned}
& +|\omega|^{2}\left(\frac{e^{-\Gamma_{S} \Delta \tau}}{\Gamma_{S}}+\frac{e^{-\Gamma_{L} \Delta \tau}}{\Gamma_{L}}\right)+\frac{2 \gamma}{\Delta \Gamma}\left(\frac{e^{-\Gamma_{L} \Delta \tau}}{\Gamma_{L}}-\frac{e^{-\Gamma_{S} \Delta \tau}}{\Gamma_{S}}\right) \\
& +\frac{2 \alpha}{\Delta m} e^{-(\bar{\Gamma}+\alpha-\gamma) \Delta \tau} \frac{((\bar{\Gamma}+\alpha-\gamma) \sin (\Delta m \Delta \tau)+\Delta m \cos (\Delta m \Delta \tau))}{(\bar{\Gamma}+\alpha-\gamma)^{2}+\Delta m^{2}} \\
& \pm \frac{4}{\Delta m^{2}+(\bar{\Gamma}+\alpha-\gamma)^{2}+2(\bar{\Gamma}+\alpha-\gamma) \Gamma_{S}+\Gamma_{S}^{2}} \\
& \times\left(\frac{2 \beta}{|d|} e^{-\Delta \tau \Gamma_{S}}\left(\Delta m \cos \left(\phi_{S W}\right)-\left(\bar{\Gamma}+\alpha-\gamma+\Gamma_{S}\right) \sin \left(\phi_{S W}\right)\right)\right) \\
& +|\omega| e^{-\Delta \tau \Gamma_{S}}\left(\Delta m \sin (\Omega)+\left(\bar{\Gamma}+\alpha-\gamma+\Gamma_{S}\right) \cos (\Omega)\right) \\
& +\frac{2 \beta}{|d|} e^{-(\bar{\Gamma}+\alpha-\gamma) \Delta \tau}\left(\Delta m \cos \left(\Delta m \Delta \tau-\phi_{S W}\right)+\left(\bar{\Gamma}+\alpha-\gamma+\Gamma_{S}\right) \sin \left(\Delta m \Delta \tau-\phi_{S W}\right)\right) \\
& \left.+|\omega| e^{-(\bar{\Gamma}+\alpha-\gamma) \Delta \tau}\left(-\Delta m \sin (\Delta m \Delta \tau-\Omega)+\left(\bar{\Gamma}+\alpha-\gamma+\Gamma_{S}\right) \cos (\Delta m \Delta \tau-\Omega)\right)\right) \\
& \pm \frac{4}{\Delta m^{2}+(\bar{\Gamma}+\alpha-\gamma)^{2}+2(\bar{\Gamma}+\alpha-\gamma) \Gamma_{L}+\Gamma_{L}^{2}} \\
& \times\left(\frac{2 \beta}{|d|} e^{-\Delta \tau \Gamma_{L}}\left(\Delta m \cos \left(\phi_{S W}\right)+\left(\bar{\Gamma}+\alpha-\gamma+\Gamma_{L}\right) \sin \left(\phi_{S W}\right)\right)\right. \\
& +|\omega| e^{-\Delta \tau \Gamma_{L}}\left(-\Delta m \sin (\Omega)+\left(\bar{\Gamma}+\alpha-\gamma+\Gamma_{L}\right) \cos (\Omega)\right) \\
& +\frac{2 \beta}{|d|} e^{-(\bar{\Gamma}+\alpha-\gamma) \Delta \tau}\left(\Delta m \cos \left(\Delta m \Delta \tau-\phi_{S W}\right)+\left(\bar{\Gamma}+\alpha-\gamma+\Gamma_{L}\right) \sin \left(\Delta m \Delta \tau-\phi_{S W}\right)\right) \\
& \left.\left.+|\omega| e^{-(\bar{\Gamma}+\alpha-\gamma) \Delta \tau}\left(-\Delta m \sin (\Delta m \Delta \tau+\Omega)+\left(\bar{\Gamma}+\alpha-\gamma+\Gamma_{L}\right) \cos (\Delta m \Delta \tau+\Omega)\right)\right)\right]
\end{aligned}
$$

We plot the corresponding integrated double-decay rate for $\ell^{+} \ell^{+}$in Fig. 3.

Next we consider the case in which one of the Kaons decays to $\ell^{+}$and the other to $\ell^{-}$. On noting that the relevant observable corresponding to the $\ell^{-}$case is associated with the operator $\mathcal{O}_{l-}$ of (3.1):

$$
\mathcal{O}_{l-}=\frac{|a|^{2}}{2}\left(\begin{array}{cc}
1 & -1 \\
-1 & 1
\end{array}\right)
$$

the relevant double-decay rate reads:

$$
\begin{aligned}
\mathcal{P}\left(l^{+}, \tau_{1} ; l^{-}, \tau_{2}\right)= & \frac{a^{4}}{8}\left[(1+4 \operatorname{Re}(\Delta-\beta / d)) e^{-\Gamma_{L} \tau_{2}-\Gamma_{S} \tau_{1}}+(1+4 \operatorname{Re}(\beta / d-\Delta)) e^{-\Gamma_{L} \tau_{1}-\Gamma_{S} \tau_{2}}\right. \\
& +2(\cos (\Delta m \Delta \tau)+4 \operatorname{Im}(\Delta+\beta / d) \sin (\Delta m \Delta \tau)) e^{-(\bar{\Gamma}+\alpha-\gamma)\left(\tau_{1}+\tau_{2}\right)} \\
& +\left(2|\omega|^{2} \cos \left(\Delta m\left(\tau_{1}+\tau_{2}\right)\right)-\frac{2 \alpha}{\Delta m} \sin \left(\Delta m\left(\tau_{1}+\tau_{2}\right)\right)\right) e^{-(\bar{\Gamma}+\alpha-\gamma)\left(\tau_{1}+\tau_{2}\right)} \\
& +\left(\left(|\omega|^{2}-\frac{2 \gamma}{\Delta \Gamma}\right) e^{-\Gamma_{S}\left(\tau_{1}+\tau_{2}\right)}+\left(|\omega|^{2}+\frac{2 \gamma}{\Delta \Gamma}\right) e^{-\Gamma_{L}\left(\tau_{1}+\tau_{2}\right)}\right)
\end{aligned}
$$



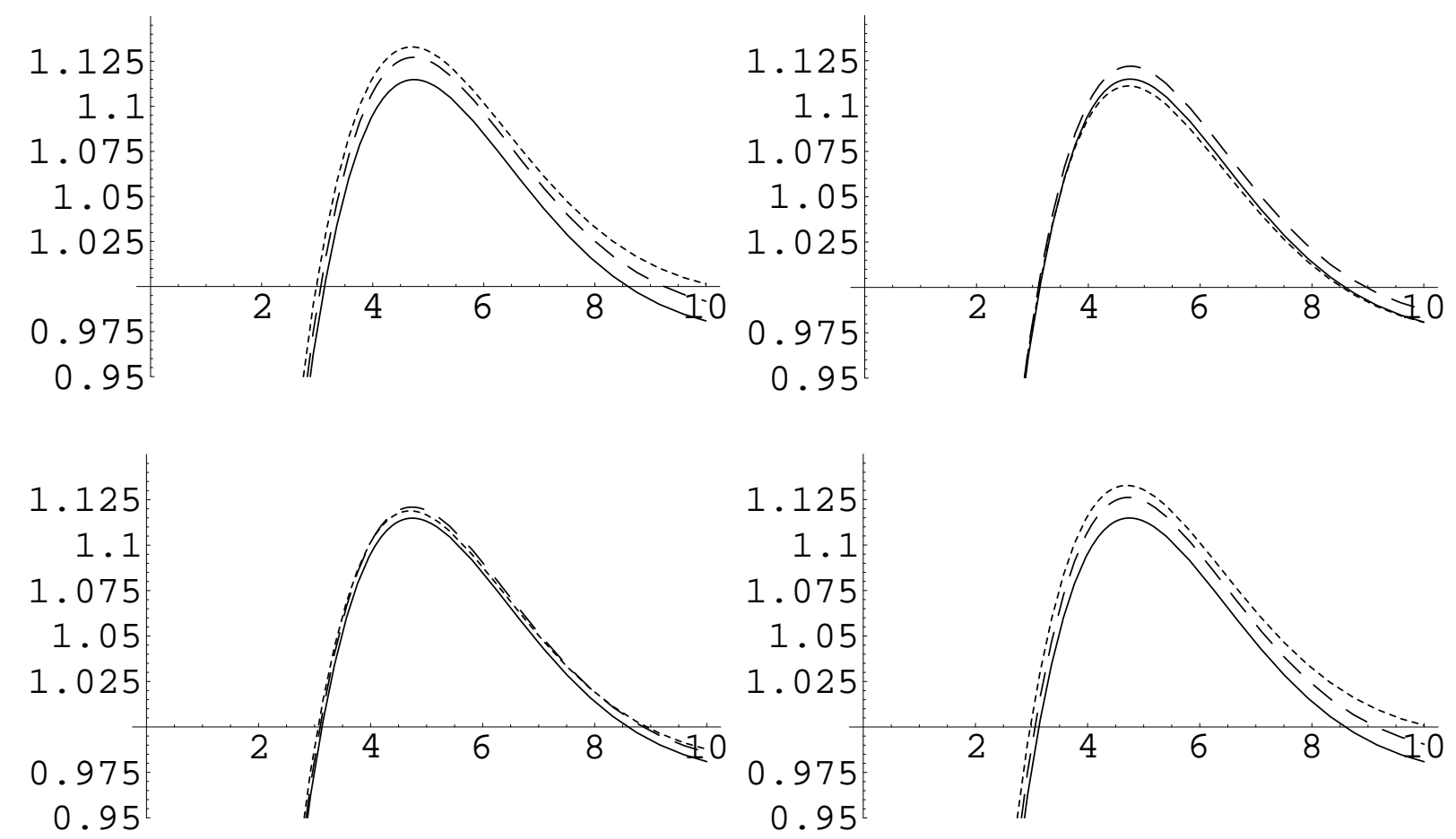

FIG. 3: Plots of $\overline{\mathcal{P}}\left(l^{+}, l^{+}, \Delta \tau\right)$ (in units of $\Delta \tau a^{4} / 4$ vs. $\Delta \tau$ with $\alpha, \beta, \gamma, \omega$ non zero as dashed curve and $\alpha, \beta, \gamma, \omega=0$ is the solid curve, and the long-dashed curve denotes the case where only $\omega \neq 0$.

$$
\begin{aligned}
& +\left(\frac{4 \beta}{|d|} \sin \left(\Delta m \tau_{1}-\phi_{S W}\right)+2|\omega| \cos \left(\Delta m \tau_{1}-\Omega\right)\right) e^{-(\bar{\Gamma}+\alpha-\gamma) \tau_{1}} e^{-\Gamma_{S} \tau_{2}} \\
& -\left(\frac{4 \beta}{|d|} \sin \left(\Delta m \tau_{2}-\phi_{S W}\right)-2|\omega| \cos \left(\Delta m \tau_{2}-\Omega\right)\right) e^{-(\bar{\Gamma}+\alpha-\gamma) \tau_{2}} e^{-\Gamma_{S} \tau_{1}} \\
& +\left(\frac{4 \beta}{|d|} \sin \left(\Delta m \tau_{1}+\phi_{S W}\right)+2|\omega| \cos \left(\Delta m \tau_{1}+\Omega\right)\right) e^{-(\bar{\Gamma}+\alpha-\gamma) \tau_{1}} e^{-\Gamma_{L} \tau_{2}} \\
& \left.-\left(\frac{4 \beta}{|d|} \sin \left(\Delta m \tau_{2}+\phi_{S W}\right)-2|\omega| \cos \left(\Delta m \tau_{2}+\Omega\right)\right) e^{-(\bar{\Gamma}+\alpha-\gamma) \tau_{2}} e^{-\Gamma_{L} \tau_{1}}\right]
\end{aligned}
$$

and the integrated over time distribution is:

$$
\begin{aligned}
\overline{\mathcal{P}} & \left(l^{+} ; l^{-}, \Delta \tau\right)=\frac{a^{4}}{16}\left[(1+4 \operatorname{Re}(\Delta-\beta / d)) \frac{2 e^{-\Gamma_{L} \Delta \tau}}{\Gamma_{S}+\Gamma_{L}}+(1+4 \operatorname{Re}(\beta / d-\Delta)) \frac{2 e^{-\Gamma_{S} \Delta \tau}}{\Gamma_{S}+\Gamma_{L}}\right. \\
+ & 2(\cos (\Delta m \Delta \tau)+4 \operatorname{Im}(\Delta+\beta / d) \sin (\Delta m \Delta \tau)) \frac{e^{-(\bar{\Gamma}+\alpha-\gamma) \Delta \tau}}{(\bar{\Gamma}+\alpha-\gamma)} \\
+ & \frac{e^{-(\bar{\Gamma}+\alpha-\gamma) \Delta \tau}}{(\bar{\Gamma}+\alpha-\gamma)+\Delta m^{2}}\left(2|\omega|^{2}((\bar{\Gamma}+\alpha-\gamma) \cos (\Delta m \Delta \tau)-\Delta m \sin (\Delta m \Delta \tau))\right. \\
& \left.-\frac{2 \alpha}{\Delta m}(\Delta m \cos (\Delta m \Delta \tau)+(\bar{\Gamma}+\alpha-\gamma) \sin (\Delta m \Delta \tau))\right)
\end{aligned}
$$




$$
\begin{aligned}
& +\left(\left(|\omega|^{2}-\frac{2 \gamma}{\Delta \Gamma}\right) \frac{e^{-\Gamma_{S} \Delta \tau}}{\Gamma_{S}}+\left(|\omega|^{2}+\frac{2 \gamma}{\Delta \Gamma}\right) \frac{e^{-\Gamma_{L} \Delta \tau}}{\Gamma_{L}}\right) \\
& +\frac{4}{\Delta m^{2}+(\bar{\Gamma}+\alpha-\gamma)^{2}+2(\bar{\Gamma}+\alpha-\gamma) \Gamma_{S}+\Gamma_{S}^{2}} \\
& \times\left(\frac{2 \beta}{|d|} e^{-\Delta \tau \Gamma_{S}}\left(\Delta m \cos \left(\phi_{S W}\right)-\left(\bar{\Gamma}+\alpha-\gamma+\Gamma_{S}\right) \sin \left(\phi_{S W}\right)\right)\right. \\
& +|\omega| e^{-\Delta \tau \Gamma_{S}}\left(\Delta m \sin (\Omega)+\left(\bar{\Gamma}+\alpha-\gamma+\Gamma_{S}\right) \cos (\Omega)\right) \\
& -\frac{2 \beta}{|d|} e^{-(\bar{\Gamma}+\alpha-\gamma) \Delta \tau}\left(\Delta m \cos \left(\Delta m \Delta \tau-\phi_{S W}\right)+\left(\bar{\Gamma}+\alpha-\gamma+\Gamma_{S}\right) \sin \left(\Delta m \Delta \tau-\phi_{S W}\right)\right) \\
& \left.-|\omega| e^{-(\bar{\Gamma}+\alpha-\gamma) \Delta \tau}\left(-\Delta m \sin (\Delta m \Delta \tau-\Omega)+\left(\bar{\Gamma}+\alpha-\gamma+\Gamma_{S}\right) \cos (\Delta m \Delta \tau-\Omega)\right)\right) \\
& +\frac{4}{\Delta m^{2}+(\bar{\Gamma}+\alpha-\gamma)^{2}+2(\bar{\Gamma}+\alpha-\gamma) \Gamma_{L}+\Gamma_{L}^{2}} \\
& \times\left(\frac{2 \beta}{|d|} e^{-\Delta \tau \Gamma_{L}}\left(\Delta m \cos \left(\phi_{S W}\right)+\left(\bar{\Gamma}+\alpha-\gamma+\Gamma_{L}\right) \sin \left(\phi_{S W}\right)\right)\right. \\
& +|\omega| e^{-\Delta \tau \Gamma_{L}}\left(-\Delta m \sin (\Omega)+\left(\bar{\Gamma}+\alpha-\gamma+\Gamma_{L}\right) \cos (\Omega)\right) \\
& -\frac{2 \beta}{|d|} e^{-(\bar{\Gamma}+\alpha-\gamma) \Delta \tau}\left(\Delta m \cos \left(\Delta m \Delta \tau-\phi_{S W}\right)+\left(\bar{\Gamma}+\alpha-\gamma+\Gamma_{L}\right) \sin \left(\Delta m \Delta \tau-\phi_{S W}\right)\right) \\
& \left.\left.+|\omega| e^{-(\bar{\Gamma}+\alpha-\gamma) \Delta \tau}\left(-\Delta m \sin (\Delta m \Delta \tau+\Omega)+\left(\bar{\Gamma}+\alpha-\gamma+\Gamma_{L}\right) \cos (\Delta m \Delta \tau+\Omega)\right)\right)\right]
\end{aligned}
$$

We next give the three pion decay channels using the relevant observable (3.1),

$$
\mathcal{O}_{3 \pi}=\left|X_{3 \pi}\right|^{2}\left(\begin{array}{cc}
\left|Y_{3 \pi}\right|^{2} & Y_{3 \pi}^{*} \\
Y_{3 \pi} & 1
\end{array}\right) \text {. }
$$

Here we take $\left|Y_{3 \pi}\right|=0$, since we ignore the associated CP and CPT violation in the decay and obtain:

$$
\mathcal{O}_{3 \pi}=\left|X_{3 \pi}\right|^{2}\left(\begin{array}{ll}
0 & 0 \\
0 & 1
\end{array}\right) .
$$

This leads to the following expression for the associated double-time distribution

$$
\begin{aligned}
\mathcal{P} & \left(3 \pi, \tau_{1} ; 3 \pi, \tau_{2}\right)=\frac{\left|X_{3 \pi}\right|^{4}}{2}\left[R_{S} e^{-\Gamma_{L} \tau_{2}-\Gamma_{S} \tau_{1}}+R_{S} e^{-\Gamma_{L} \tau_{1}-\Gamma_{S} \tau_{2}}\right. \\
& -2\left|\bar{\eta}_{3 \pi}\right|^{2} \cos \left(\Delta m\left(\tau_{1}-\tau_{2}\right)\right) e^{-(\bar{\Gamma}+\alpha-\gamma)\left(\tau_{1}+\tau_{2}\right)}+\left(|\omega|^{2}+\frac{2 \gamma}{\Delta \Gamma}+\frac{4 \beta}{|d|}\left|\bar{\eta}_{3 \pi}\right| \frac{\sin \phi_{3 \pi}}{\cos \phi_{S W}}\right) e^{-\Gamma_{L}\left(\tau_{1}+\tau_{2}\right)} \\
& +\left|\bar{\eta}_{3 \pi}\right| e^{-\Gamma_{L} \tau_{1}-(\bar{\Gamma}+\alpha-\gamma) \tau_{2}}\left(-2|\omega| \cos \left(\Delta m \tau_{2}-\phi_{3 \pi}+\Omega\right)+\frac{4 \beta}{|d|} \sin \left(\Delta m \tau_{2}-\phi_{3 \pi}+\phi_{S W}\right)\right) \\
& \left.+\left|\bar{\eta}_{3 \pi}\right| e^{-\Gamma_{L} \tau_{2}-(\bar{\Gamma}+\alpha-\gamma) \tau_{1}}\left(2|\omega| \cos \left(\Delta m \tau_{1}-\phi_{3 \pi}+\Omega\right)+\frac{4 \beta}{|d|} \sin \left(\Delta m \tau_{1}-\phi_{3 \pi}+\phi_{S W}\right)\right)\right],
\end{aligned}
$$



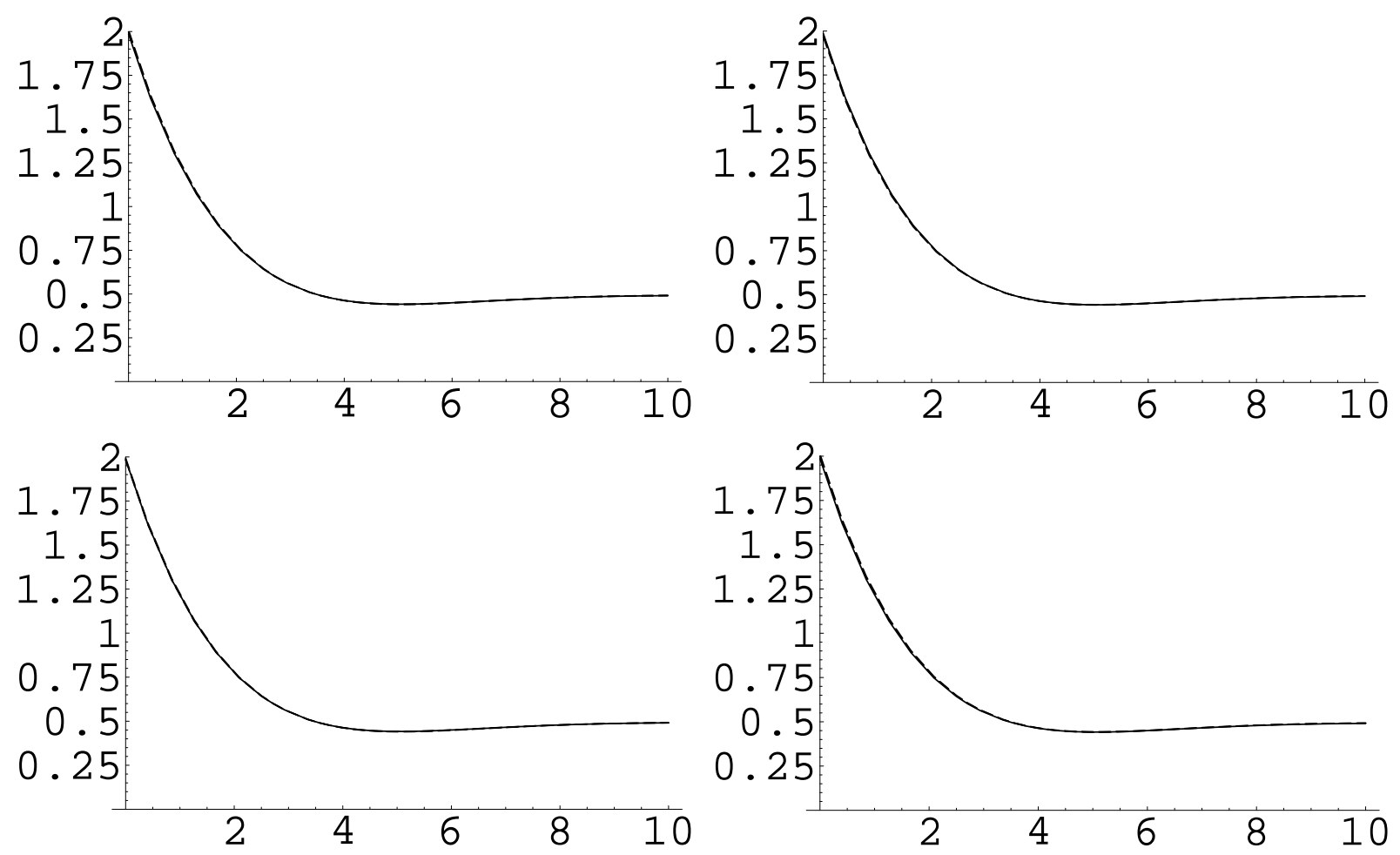

FIG. 4: Plots of $\overline{\mathcal{P}}\left(l^{+} ; l^{-}, \Delta \tau\right)$, in units of $\tau_{S} \frac{|a|^{4}}{4}$, vs. $\Delta \tau$ with $\alpha, \beta, \gamma, \omega$ non zero as dashed curve and $\alpha, \beta, \gamma, \omega=0$ is the solid curve, and the long-dashed curve denotes the case where only $\omega \neq 0$.

which integrated over time gives

$$
\begin{aligned}
\overline{\mathcal{P}}\left(3 \pi, \tau_{1} ; 3\right. & \left.3 \pi, \tau_{2}\right)=\frac{\left|X_{3 \pi}\right|^{4}}{4}\left[\frac{2 R_{S}\left(e^{-\Gamma_{L} \Delta \tau}+e^{-\Gamma_{S} \Delta \tau}\right)}{\Gamma_{S}+\Gamma_{L}}\right. \\
- & 2\left|\bar{\eta}_{3 \pi}\right|^{2} \cos (\Delta m \Delta \tau) \frac{e^{-(\bar{\Gamma}+\alpha-\gamma) \Delta \tau}}{\bar{\Gamma}+\alpha-\gamma}+\left(|\omega|^{2}+\frac{2 \gamma}{\Delta \Gamma}+\frac{4 \beta}{|d|}\left|\bar{\eta}_{3 \pi}\right| \frac{\sin \phi_{3 \pi}}{\cos \phi_{S W}}\right) \frac{e^{-\Gamma_{L} \Delta \tau}}{\Gamma_{L}} \\
+ & \frac{4\left|\bar{\eta}_{3 \pi}\right| e^{-(\bar{\Gamma}+\alpha-\gamma) \Delta \tau}}{\Delta m^{2}+(\bar{\Gamma}+\alpha-\gamma)^{2}+2(\bar{\Gamma}+\alpha-\gamma) \Gamma_{L}+\Gamma_{L}^{2}} \\
\times & \left(| \omega | e ^ { - ( \overline { \Gamma } + \alpha - \gamma ) \Delta \tau } \left(-\left(\bar{\Gamma}+\alpha-\gamma+\Gamma_{L}\right) \cos \left(\Delta m \Delta \tau-\phi_{3 \pi}+\Omega\right)\right.\right. \\
& \left.+\Delta m \sin \left(\Delta m \Delta \tau-\phi_{3 \pi}+\Omega\right)\right) \\
+ & \frac{2 \beta}{|d|} e^{-(\bar{\Gamma}+\alpha-\gamma) \Delta \tau}\left(\left(\bar{\Gamma}+\alpha-\gamma+\Gamma_{L}\right) \sin \left(\Delta m \Delta \tau-\phi_{3 \pi}+\phi_{S W}\right)\right. \\
& \left.+\Delta m \cos \left(\Delta m \Delta \tau-\phi_{3 \pi}+\phi_{S W}\right)\right) \\
+ & |\omega| e^{-\Gamma_{L} \Delta \tau}\left(\left(\bar{\Gamma}+\alpha-\gamma+\Gamma_{L}\right) \cos \left(\Omega-\phi_{3 \pi}\right)-\Delta m \sin \left(\Omega-\phi_{3 \pi}\right)\right) \\
+ & \left.\left.\frac{2 \beta}{|d|} e^{-\Gamma_{L} \Delta \tau}\left(\left(\bar{\Gamma}+\alpha-\gamma+\Gamma_{L}\right) \sin \left(\phi_{S W}-\phi_{3 \pi}\right)+\Delta m \cos \left(\phi_{S W}-\phi_{3 \pi}\right)\right)\right)\right]
\end{aligned}
$$



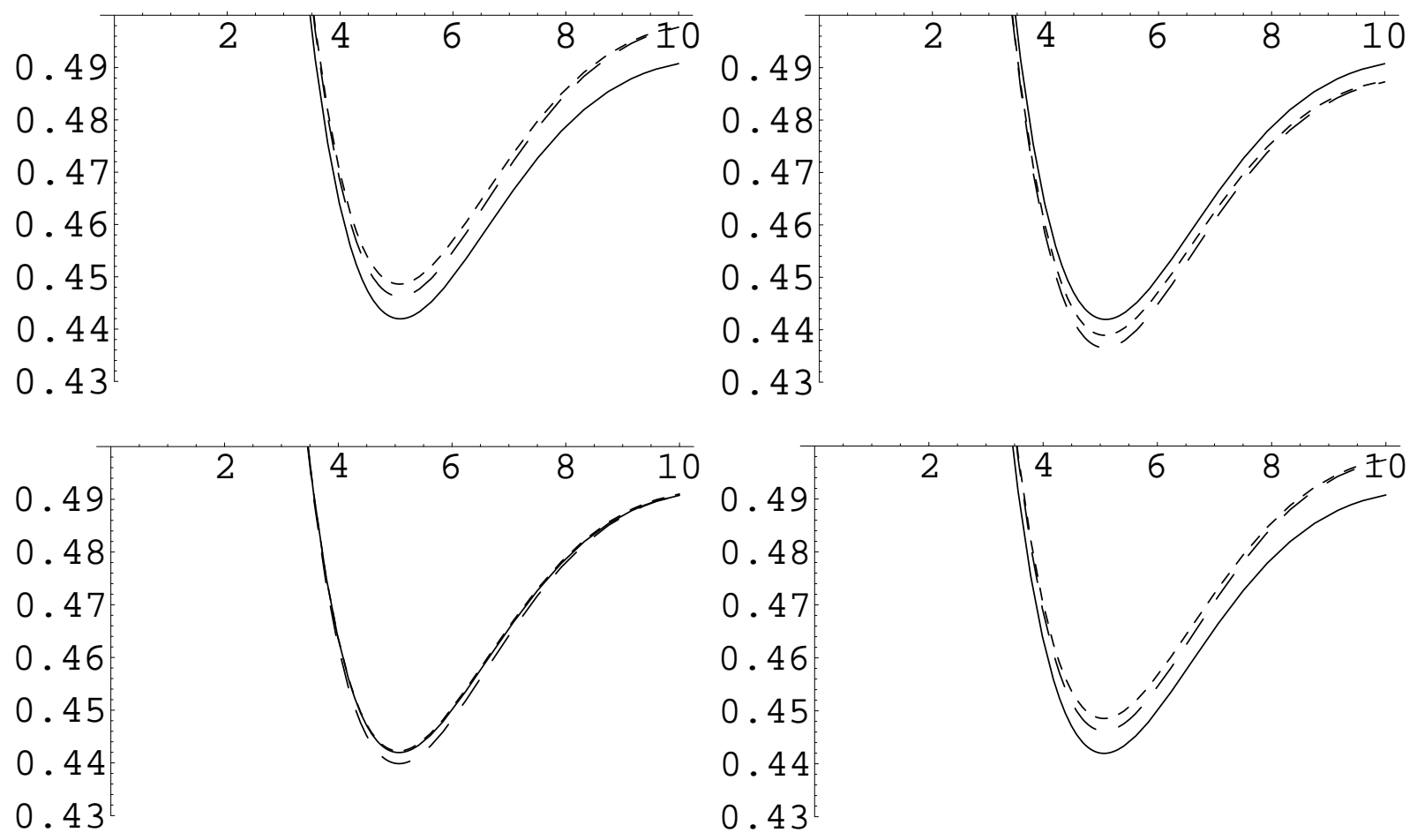

FIG. 5: As in Fig. 4, but shown in detail to better illustrate the difference between the various cases. Again, the dashed curves are with $\alpha, \beta, \gamma, \omega$ all non-zero, the long-dashed curve has only $\omega \neq 0$, whilst the solid curve represents $\alpha, \beta, \gamma, \omega=0$.

We plot this function vs. $\Delta \tau$ in Fig. 6. We use units of $\tau_{L}$ for convenience. The quantummechanical case with $\omega=\alpha=\beta=\gamma=0$ coincides with the horizontal axis. As we observe from the graph, the CPTV and decoherence effects are pronounced near $\Delta \tau=0$. Thus, in principle this is the cleanest way of bounding such effects, but unfortunately in practice this is a very difficult channel to measure experimentally.

This completes the analysis of identical-final-states observables.

\section{B. General Final States}

We consider in this subsection observables for the case where the final states are different.

We first consider the case in which one Kaon decays to $\pi^{+} \pi^{-}$and the other to $\pi^{0} \pi^{0}$. For the double-decay rate we find

$$
\mathcal{P}\left(\pi^{+} \pi^{-}, \tau_{1} ; \pi^{0} \pi^{0}, \tau_{2}\right)=2 A_{0}^{4}\left[R_{L}^{00} e^{-\Gamma_{L} \tau_{2}-\Gamma_{S} \tau_{1}}+R_{L}^{+-} e^{-\Gamma_{L} \tau_{1}-\Gamma_{S} \tau_{2}}\right.
$$




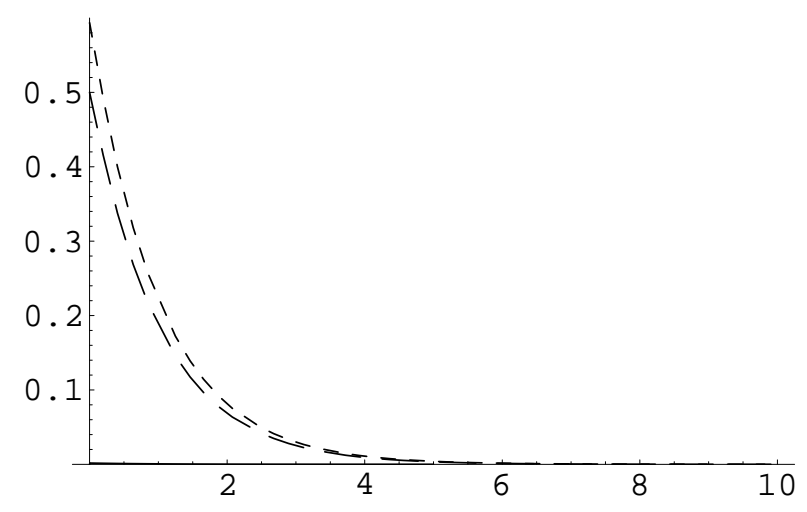

FIG. 6: Plots of $\overline{\mathcal{P}}(3 \pi ; 3 \pi, \Delta \tau)$, in units of $\tau_{L}\left|\bar{\eta}_{+-}\right|^{2} \frac{\left|X_{3 \pi}\right|^{4}}{2}$ (for convenience), vs. $\Delta \tau$ (also in units of $\left.\tau_{L}\right)$, with $\alpha, \beta, \gamma, \omega$ non zero as dashed curve and $\alpha, \beta, \gamma, \omega=0$ is the solid curve, and the longdashed curve denotes the case where only $|\omega|=\left|\bar{\eta}_{+-}\right| \neq 0$ (this value is chosen for concreteness). We see that the CPTV effects are pronounced near the origin and are easily disentangled from the quantum mechanical case (which coincides with the horizontal axis).

$$
\begin{aligned}
& -2\left|\bar{\eta}_{+-}\right|\left|\bar{\eta}_{00}\right| \cos \left(\Delta m\left(\tau_{1}-\tau_{2}\right)+\phi_{+-}-\phi_{00}\right) e^{-(\bar{\Gamma}+\alpha-\gamma)\left(\tau_{1}+\tau_{2}\right)} \\
& +\left(|\omega|^{2}-\frac{2 \gamma}{\Delta \Gamma}-\frac{2 \beta}{|d|} \frac{\left(\left|\bar{\eta}_{+-}\right| \sin \phi_{+-}+\left|\bar{\eta}_{00}\right| \sin \phi_{00}\right)}{\cos \phi_{S W}}\right) e^{-\Gamma_{S}\left(\tau_{1}+\tau_{2}\right)} \\
& +\left|\bar{\eta}_{+-}\right| e^{-\Gamma_{S} \tau_{2}-(\bar{\Gamma}+\alpha-\gamma) \tau_{1}}\left(2|\omega| \cos \left(\Delta m \tau_{1}+\phi_{+-}-\Omega\right)+\frac{4 \beta}{|d|} \sin \left(\Delta m \tau_{1}+\phi_{+-}-\phi_{S W}\right)\right) \\
& \left.+\left|\bar{\eta}_{00}\right| e^{-\Gamma_{S} \tau_{1}-(\bar{\Gamma}+\alpha-\gamma) \tau_{2}}\left(-2|\omega| \cos \left(\Delta m \tau_{2}+\phi_{00}-\Omega\right)+\frac{4 \beta}{|d|} \sin \left(\Delta m \tau_{2}+\phi_{00}-\phi_{S W}\right)\right)\right] .
\end{aligned}
$$

The integrated-over-time distribution is given by

$$
\begin{aligned}
\overline{\mathcal{P}} & \left(\pi^{+} \pi^{-} ; \pi^{0} \pi^{0} ; \Delta \tau\right)=2 A_{0}^{4}\left[R_{L}^{00} \frac{e^{-\Gamma_{L} \Delta \tau}}{\Gamma_{L}+\Gamma_{S}}+R_{L}^{+-} \frac{e^{-\Gamma_{S} \Delta \tau}}{\Gamma_{L}+\Gamma_{S}}\right. \\
- & \left|\bar{\eta}_{+-}\right|\left|\bar{\eta}_{00}\right| \cos \left(\Delta m \Delta \tau+\phi_{+-}-\phi_{00}\right) \frac{e^{-(\bar{\Gamma}+\alpha-\gamma) \Delta \tau}}{\bar{\Gamma}+\alpha-\gamma} \\
& +\left(|\omega|^{2}-\frac{2 \gamma}{\Delta \Gamma}-\frac{2 \beta}{|d|} \frac{\left(\left|\bar{\eta}_{+-}\right| \sin \phi_{+-}+\left|\bar{\eta}_{00}\right| \sin \phi_{00}\right)}{\cos \phi_{S W}}\right) \frac{e^{-\Gamma_{S} \Delta \tau}}{2 \Gamma_{S}} \\
& +\frac{2 \quad(\bar{\Gamma}+\alpha-\gamma) \Gamma_{S}+\Gamma_{S}^{2}}{\Delta m^{2}+(\bar{\Gamma}+\alpha-\gamma)^{2}+2(\bar{\Gamma}} \\
& \times\left[| \overline { \eta } _ { + - } | e ^ { - \Gamma _ { S } \Delta \tau } \left(|\omega|\left(\left(\bar{\Gamma}+\alpha-\gamma+\Gamma_{S}\right) \cos \left(\phi_{+-}-\Omega\right)-\Delta m \sin \left(\phi_{+-}-\Omega\right)\right)\right.\right. \\
& \left.+\frac{2 \beta}{|d|}\left(\left(\bar{\Gamma}+\alpha-\gamma+\Gamma_{S}\right) \sin \left(\phi_{+-}-\phi_{S W}\right)+\Delta m \cos \left(\phi_{+-}-\phi_{S W}\right)\right)\right) \\
& +\left|\bar{\eta}_{00}\right| e^{-(\bar{\Gamma}+\alpha-\gamma) \Delta \tau}\left(|\omega|\left(-\left(\bar{\Gamma}+\alpha-\gamma+\Gamma_{S}\right) \cos \left(\Delta m \Delta \tau+\phi_{00}-\Omega\right)+\Delta m \sin \left(\Delta m \Delta \tau+\phi_{00}-\Omega\right)\right)\right.
\end{aligned}
$$



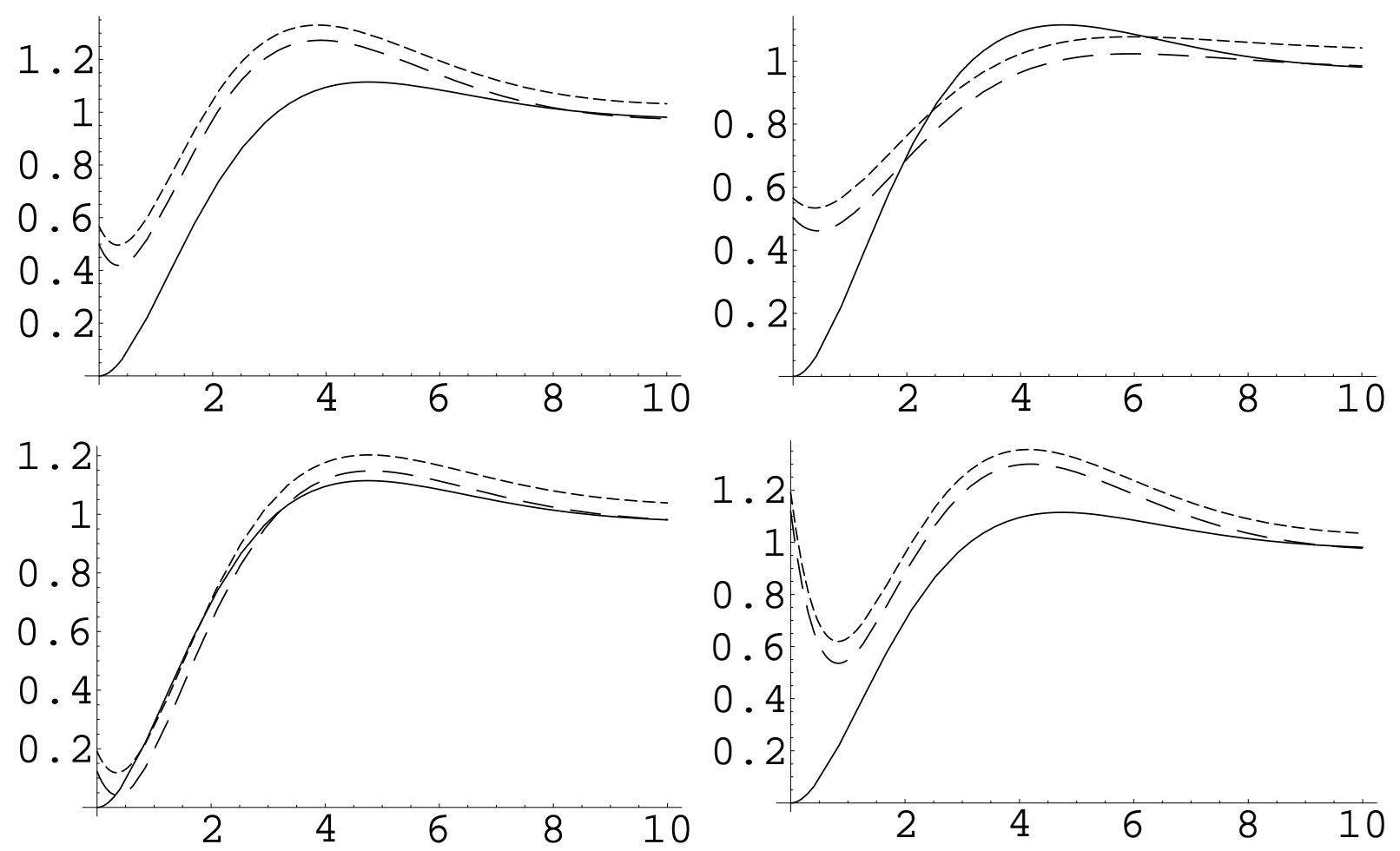

FIG. 7: Plots of $\overline{\mathcal{P}}\left(\pi^{+} \pi^{-} ; \pi^{0} \pi^{0} ; \Delta \tau\right)$, in units of $\tau_{S}\left|\bar{\eta}_{+-}\right|^{2} \frac{\left|X_{+-}\right|^{2}\left|X_{00}\right|^{2}}{2}$, vs. $\Delta \tau$ with $\alpha, \beta, \gamma, \omega$ non zero as dashed curve and $\alpha, \beta, \gamma, \omega=0$ is the solid curve, and the long-dashed curve denotes the case where only $\omega \neq 0$.

$$
\left.\left.\left.+\frac{2 \beta}{|d|}\left(\bar{\Gamma}+\alpha-\gamma+\Gamma_{S}\right)\left(\sin \left(\Delta m \Delta \tau+\phi_{00}-\phi_{S W}\right)+\Delta m \cos \left(\Delta m \Delta \tau+\phi_{00}-\phi_{S W}\right)\right)\right)\right]\right]
$$

Finally we consider the case in which one of the final states consists of two pions, and the other is the result of a kaon semileptonic decay. The relevant double-decay rate is given by:

$$
\begin{aligned}
\mathcal{P} & \left(\pi^{ \pm}, \tau_{1} ; l^{ \pm}, \tau_{2}\right)=\frac{A_{0}^{2} a^{2}}{4}\left[\left(1 \pm \delta_{L}+\frac{\gamma}{\Delta \Gamma}\right) e^{-\Gamma_{L} \tau_{2}-\Gamma_{S} \tau_{1}}+R_{L} e^{-\Gamma_{L} \tau_{1}-\Gamma_{S} \tau_{2}}\right. \\
& \mp 2\left|\bar{\eta}_{+-}\right| \cos \left(\Delta m\left(\tau_{1}-\tau_{2}\right)+\phi_{+-}\right) e^{-(\bar{\Gamma}+\alpha-\gamma)\left(\tau_{1}+\tau_{2}\right)}+\left(|\omega|^{2}-\frac{2 \gamma}{\Delta \Gamma}-\frac{4 \beta\left|\bar{\eta}_{+-}\right|}{|d|} \frac{\sin \phi_{+-}}{\cos \phi_{S W}}\right) e^{-\Gamma_{S}\left(\tau_{1}+\tau_{2}\right)} \\
& \left. \pm\left(\frac{4 \beta}{|d|} \sin \left(\Delta m \tau_{2}-\phi_{S W}\right)-2|\omega| \cos \left(\Delta m \tau_{2}-\Omega\right)\right) e^{-\Gamma_{S} \tau_{1}-(\bar{\Gamma}+\alpha-\gamma) \tau_{2}}\right]
\end{aligned}
$$

where we used the definition $\delta_{L}=2 \operatorname{Re} \epsilon_{L}^{+}$. 

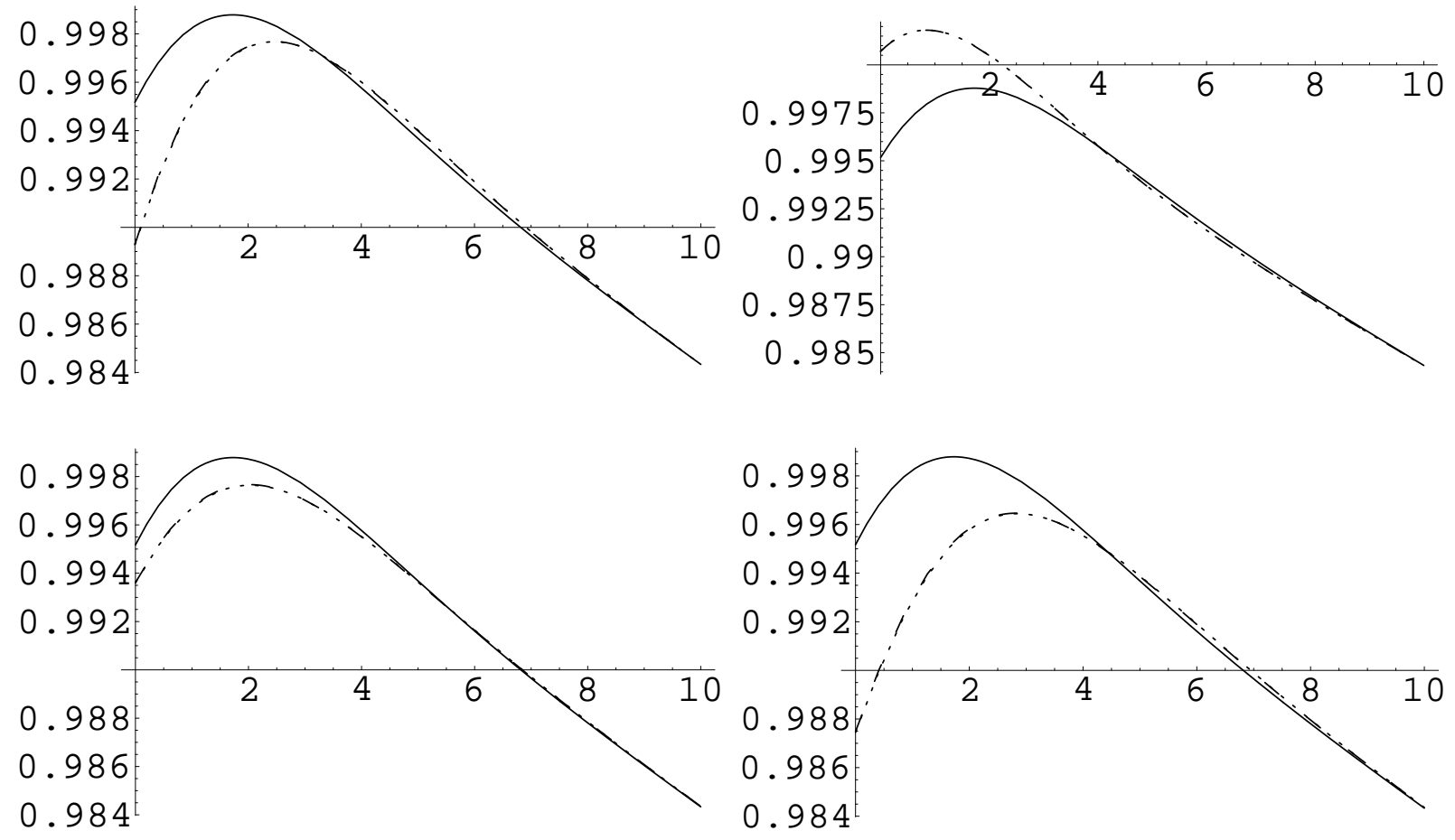

FIG. 8: Plots of $\overline{\mathcal{P}}\left(\pi^{ \pm} ; l^{ \pm}, \Delta \tau\right)$, in units of $\tau_{S} \frac{\left|X_{+-}\right|^{2}|a|^{2}}{8}$, vs. $\Delta \tau$ with $\alpha, \beta, \gamma, \omega$ non zero as dashed curve and $\alpha, \beta, \gamma, \omega=0$ is the solid curve, and the long-dashed curve denotes the case where only $\omega \neq 0$. Notice that in the scale chosen, the dashed and long-dashed curves overlap completely.

The integrated double-decay rate reads:

$$
\begin{aligned}
& \overline{\mathcal{P}}\left(\pi^{ \pm} ; l^{ \pm}, \Delta \tau\right)=\frac{A_{0}^{2} a^{2}}{4}\left[\left(1 \pm \delta_{L}+\frac{\gamma}{\Delta \Gamma}\right) \frac{e^{-\Gamma_{L} \Delta \tau}}{\Gamma_{L}+\Gamma_{S}}+R_{L} \frac{e^{-\Gamma_{S} \Delta \tau}}{\Gamma_{L}+\Gamma_{S}}\right. \\
& \mp\left|\bar{\eta}_{+-}\right| \cos \left(\Delta m \Delta \tau+\phi_{+-}\right) \frac{e^{-(\bar{\Gamma}+\alpha-\gamma) \Delta \tau}}{(\bar{\Gamma}+\alpha-\gamma)}+\left(|\omega|^{2}-\frac{2 \gamma}{\Delta \Gamma}-\frac{4 \beta\left|\bar{\eta}_{+-}\right|}{|d|} \frac{\sin \phi_{+-}}{\cos \phi_{S W}}\right) \frac{e^{-\Gamma_{S} \Delta \tau}}{2 \Gamma_{S}} \\
& \quad \pm \frac{2}{\Delta m^{2}+(\bar{\Gamma}+\alpha-\gamma)^{2}+2(\bar{\Gamma}+\alpha-\gamma) \Gamma_{S}+\Gamma_{S}^{2}} \\
&+\left(\frac{2 \beta}{|d|} e^{-(\bar{\Gamma}+\alpha-\gamma) \Delta \tau}\left(\Delta m \cos \left(\Delta m \Delta \tau-\phi_{S W}\right)-\left(\bar{\Gamma}+\alpha-\gamma+\Gamma_{S}\right) \sin \left(\Delta m \Delta \tau-\phi_{S W}\right)\right)\right. \\
&\left.\quad-|\omega| e^{-(\bar{\Gamma}+\alpha-\gamma) \Delta \tau}\left(-\Delta m \sin (\Delta m \Delta \tau-\Omega)+\left(\bar{\Gamma}+\alpha-\gamma+\Gamma_{S}\right) \cos (\Delta m \Delta \tau-\Omega)\right)\right)
\end{aligned}
$$

This completes our analysis of observables in a $\phi$ factory that can be used as sensitive probes for tests of possible CPTV and quantum decoherence, to leading order in the small parameters parametrizing the effects. 


\section{DISCUSSION AND CONCLUSIONS}

In this work, we have embarked into a combined treatment of decoherent and intrinsic CPTV effects in a $\phi$ factory. By studying a variety of observables, involving identical as well as general final states, we have derived analytical expressions for double-decay rates and their integrated counterparts (over time $\tau_{1}+\tau_{2}$, keeping $\Delta \tau=\tau_{1}-\tau_{2}$ fixed), which we plotted as functions of $\Delta \tau$. Our analysis included $\omega$, as well as decoherence $\alpha, \beta, \gamma$ effects. We presented the results to leading order in these small parameters.

Although the pertinent formulae are algebraically rather complex, nevertheless from our general study it became evident that one may disentangle the $\omega$ from the decoherent evolution effects by looking simultaneously at different regimes of $\Delta \tau$, namely, (i) $\Delta \tau=0$, (ii) intermediate values of $\Delta \tau$ (as compared with $\tau_{S}$, which sets a convenient characteristic time scale in the problem), with emphasis on interference terms with sinusoidal time dependence, and (iii) rather large values of $\Delta \tau$, with emphasis on the exponential damping of the relevant quantities, which is affected (slowed down) by the presence of decoherence terms $\alpha-\gamma$, in an $\omega$-independent way.

In addition to these results in a $\phi$ factory, one can obtain valuable information on the decoherence parameters $\alpha, \beta$, and $\gamma$ by looking at experiments involving single neutral Kaon beams, such as CPLEAR [17], which is achieved through flavour tagging of the neutral Kaon by means of the electric charge of the pion on the complementary side. As discussed in [4], the decoherence parameters $\alpha, \beta, \gamma$ can be separately disentangled by combined studies of several kaon asymmetries, which are again time-dependent functions. Notice though that, if complete positivity of entangled state density matrices is imposed [18], then only one decoherence parameter $\gamma>0$ survives in the model of [2], which facilitates the situation enormously.

We would like at this point to make a clarification concerning the important differences between proposed experiments here, using entangled Kaon states, and some existing proposals, notably by the CPLEAR collaboration [23], to measure EPR correlations. The reader should notice that the accuracy for measuring the appropriate observables in [23] appears to be of order $10^{-1}$; however, this accuracy pertains only to an asymmetry built from $\bar{p} p \rightarrow K^{0} \bar{K}^{0}$ in CPLEAR, looking for the dilepton decay channel, between "like" $\left(K^{0} K^{0}\right.$ or $\left.\bar{K}^{0} \bar{K}^{0}\right)$ and "unlike" $\left(K^{0} \bar{K}^{0}\right.$ or $\left.\bar{K}^{0} K^{0}\right)$ decays after time evolution. As shown in our paper, 
the most interesting observable for the $\omega$-effect comes from identical decay channels, i.e., in this case the "intra" asymmetry between $K^{0} K^{0}$ and $\bar{K}^{0} \bar{K}^{0}$, which was not discussed in ref. [23]. Furthermore, our observation [8] that the channel $\left(\pi^{+} \pi^{-}\right)\left(\pi^{+} \pi^{-}\right)$is automatically enhanced by three orders of magnitude, due to the relative $\left(\omega / \eta_{+-}\right)$amplitude between the "wrong" and the "right" symmetry states suggests that even a $10^{-1}$ experimental effect would represent a $10^{-4}$ effect in $\omega$. In fact, the expectation for the channel $e^{+} e^{-} \rightarrow K^{0} \bar{K}^{0}$ in an upgraded $D A \Phi N E$ is definitely much better, anticipating an experimental effect of the order of $10^{-4}-10^{-5}$.

In our analysis we saturated the bounds for the decoherence parameters $\alpha, \beta, \gamma$ obtained from CPLEAR 17], to disentangle the $\omega$ effect by looking at decoherent evolution of observables in a $\phi$ factory. As mentioned above, the most sensitive probe appears at first sight to be the two-charged-pion channels, as a result of enhancement factors of $\bar{\eta}_{+-}$. In fact the clearest test of deviation from quantum mechanics is to look at the behaviour of the pertinent time-integrated decay rate (3.4) near the $\Delta \tau=0$ regime, where the novel CPTV effects would yield a non-zero result for the integrated asymmetry.

However, in the presence of decoherence there is a reduction of the value of the asymmetry as compared to the pure $\omega \neq 0$ unitary case of [8], by shifting the value of the asymmetry at $\Delta \tau=0$ from $\left|\omega^{2}\right|$ to $\left|\omega^{2}\right|-2 \gamma / \Delta \Gamma$ (in appropriate units). This reduction may be significant in the case where the $|\omega|$ effects are of order $\sqrt{\gamma / \Delta \Gamma}$. Of course, for a reliable order-of-magnitude estimate of the $\omega$-parameter, one needs to resort to detailed microscopic models of QG space-time foam, a task we hope to undertake in the near future. The fact that the interference terms for $\Delta \tau \neq 0$ of the asymmetry (3.4), which depend sinusoidally on $\Delta \tau$, are proportional to $|\omega|$, and they do not depend (apart from damping factors) on decoherence parameters, allows in principle for a disentanglement of $\omega$ from decoherence $(\gamma$, ...) effects. Notice also that another clear (in principle) probe of such effects would be the time-integrated observable (3.12), associated with three-pion decay channels, near $\Delta \tau=0$. Unfortunately, however, the current experimental sensitivity for this observable is low.

Before closing we would like to make a few comments on the contamination of the actual observable for the measurement of $\epsilon^{\prime}$ by decoherence [5] and intrinsic CPTV effects. The traditional observable for the measurement of $\epsilon^{\prime}$ is the asymmetry based on charged- 
pion/neutral-pion (3.14)-type observables:

$$
\begin{aligned}
& \mathcal{A}\left(\pi^{+}, \pi^{-} ; \pi^{0}, \pi^{0} ; \delta \tau\right)=\frac{\overline{\mathcal{P}}\left(\pi^{+} \pi^{-} ; \pi^{0} \pi^{0} ; \Delta \tau\right)-\overline{\mathcal{P}}\left(\pi^{0} \pi^{0} ; \pi^{+} \pi^{-} ; \Delta \tau\right)}{\overline{\mathcal{P}}\left(\pi^{+} \pi^{-} ; \pi^{0} \pi^{0} ; \Delta \tau\right)+\overline{\mathcal{P}}\left(\pi^{0} \pi^{0} ; \pi^{+} \pi^{-} ; \Delta \tau\right)} \\
& =3 \operatorname{Re} \frac{\epsilon^{\prime}}{\epsilon} \mathcal{I}_{1}-3 \operatorname{Im} \frac{\epsilon^{\prime}}{\epsilon} \mathcal{I}_{2}
\end{aligned}
$$

In particular we find

$$
\begin{aligned}
\mathcal{I}_{1}=\frac{1}{\mathcal{D}} & {\left[e^{-\Gamma_{L} \Delta \tau}\left(1+2 \frac{\beta}{|d|\left|\bar{\eta}_{+-}\right|} \sin \left(\phi_{S W}-\phi_{+-}\right)\right)\right.} \\
- & e^{-\Gamma_{S} \Delta \tau}\left(1+2 \frac{\beta}{|d|\left|\bar{\eta}_{+-}\right|}\left(\sin \left(\phi_{S W}-\phi_{+-}\right)-|z| \sin \left(\phi_{S W}-\phi_{+-}+\phi_{z}\right)\right)\right. \\
& \left.+\frac{|\omega|}{\left|\bar{\eta}_{+-}\right|}|z| \cos \left(\Omega-\phi_{+-}+\phi_{z}\right)\right) \\
+ & e^{-(\bar{\Gamma}+\alpha-\gamma) \Delta \tau}\left(2 \frac{\beta}{|d|\left|\bar{\eta}_{+-}\right|}|z| \sin \left(\Delta m \Delta \tau+\phi_{+-}-\phi_{S W}-\phi_{z}\right)\right. \\
& \left.\left.\quad-\frac{|\omega|}{\left|\bar{\eta}_{+-}\right|}|z| \cos \left(\Delta m \Delta \tau-\Omega+\phi_{+-}-\phi_{z}\right)\right)\right]
\end{aligned}
$$

$$
\begin{aligned}
\mathcal{I}_{2}=\frac{1}{\mathcal{D}} & {\left[e^{-\Gamma_{L} \Delta \tau}\left(2 \frac{\beta}{|d|\left|\bar{\eta}_{+-}\right|} \cos \left(\phi_{S W}-\phi_{+-}\right)\right)\right.} \\
- & e^{-\Gamma_{S} \Delta \tau}\left(2 \frac{\beta}{|d|\left|\bar{\eta}_{+-}\right|}\left(\cos \left(\phi_{S W}-\phi_{+-}\right)-|z| \cos \left(\phi_{S W}-\phi_{+-}+\phi_{z}\right)\right)\right. \\
& \left.+\frac{|\omega|}{\left|\bar{\eta}_{+-}\right|}|z| \sin \left(\Omega-\phi_{+-}+\phi_{z}\right)\right) \\
+ & e^{-(\bar{\Gamma}+\alpha-\gamma) \Delta \tau}\left(2 \sin (\Delta m \Delta \tau)-2 \frac{\beta}{|d|\left|\bar{\eta}_{+-}\right|}|z| \cos \left(\Delta m \Delta \tau+\phi_{+-}-\phi_{S W}-\phi_{z}\right)\right. \\
& \left.\left.\quad-\frac{|\omega|}{\left|\bar{\eta}_{+-}\right|}|z| \sin \left(\Delta m \Delta \tau+\Omega-\phi_{S W}-\phi_{z}\right)\right)\right]
\end{aligned}
$$

and

$$
\begin{aligned}
\mathcal{D}= & e^{-\Gamma_{L} \Delta \tau}\left(1+\frac{\gamma}{\Delta \Gamma\left|\bar{\eta}_{+-}\right|^{2}}+2 \frac{\beta}{|d|\left|\bar{\eta}_{+-}\right|} \frac{\sin \left(2 \phi_{S W}-\phi_{+-}\right)}{\cos \left(\phi_{S W}\right)}\right) \\
& +e^{-\Gamma_{S} \Delta \tau}\left(1+\frac{\gamma}{\Delta \Gamma\left|\bar{\eta}_{+-}\right|^{2}} \frac{\Gamma_{L}}{\Gamma_{S}}+2 \frac{\beta}{|d|\left|\bar{\eta}_{+-}\right|}\left(\frac{\sin \left(2 \phi_{S W}-\phi_{+-}\right)}{\cos \left(\phi_{S W}\right)}-2|z| \sin \left(\phi_{S W}+\phi_{z}-\phi_{+-}\right)\right)\right.
\end{aligned}
$$




$$
\begin{aligned}
& \left.+\frac{|\omega|^{2} \bar{\Gamma}}{\left|\bar{\eta}_{+-}\right|^{2} \Gamma_{S}}-2 \frac{|\omega|}{\left|\bar{\eta}_{+-}\right|}|z| \cos \left(\Omega+\phi_{z}-\phi_{+-}\right)\right) \\
& -e^{-(\bar{\Gamma}+\alpha-\gamma)}\left(2 \cos (\Delta m \Delta \tau)-4 \frac{\beta}{|d|\left|\bar{\eta}_{+-}\right|}|z| \sin \left(\Delta m \Delta \tau+\phi_{+-}-\phi_{S W}-\phi_{z}\right)\right. \\
& \left.+\frac{2|\omega|}{\left|\bar{\eta}_{+-}\right|}|z| \cos \left(\Delta m \Delta \tau+\phi_{+-}-\Omega-\phi_{z}\right)\right)
\end{aligned}
$$

where we have defined [5]: $|z| e^{i \phi_{z}}=\frac{2 \bar{\Gamma}}{\Gamma_{S}+\bar{\Gamma}+i \Delta m}$.

It therefore becomes clear that, although in conventional situations, where the foam and intrinsic CPTV $\omega$-effects are absent, the quantities $\operatorname{Re}\left(\epsilon^{\prime} / \epsilon\right)$ and $\operatorname{Im}\left(\epsilon^{\prime} / \epsilon\right)$ can be extracted from a measurement of $\mathcal{A}\left(\pi^{+}, \pi^{-} ; \pi^{0}, \pi^{0} ; \Delta \tau\right)$ by a simple two-parameter fit, in the presence of the quantum-gravity effects this is no longer true. The coefficients $\mathcal{I}_{i}, i=1,2$ are modified in such a case by decoherence [5] and $\omega$-dependent terms.

It is worthy of pointing out at this stage that the $\omega$-dependent terms in the expression for the above asymmetry are always accompanied by factors $e^{-\Gamma_{S} \Delta \tau}$. Therefore, in the limit $\Gamma_{S} \Delta \tau \gg 1$ such terms are suppressed. We remind the reader that, in this limit, in

conventional situations, one has simply that $\mathcal{A}\left(\pi^{+}, \pi^{-} ; \pi^{0}, \pi^{0} ; \Delta \tau\right) \rightarrow 3 \operatorname{Re} \frac{\epsilon^{\prime}}{\epsilon}$. In contrast, in the CPTV foamy situation the same limit contains, to leading order, terms proportional to both real and imaginary parts of $\epsilon^{\prime} / \epsilon$, with coefficients dependent on the decoherence parameters, $\beta, \gamma$, but independent of $\omega$. It is also worthy of noting the form of $\mathcal{I}_{2}$ in this limit: $\mathcal{I}_{2}=\mathcal{O}(\beta)$ to leading order in the small parameters. In complete positivity models [18], therefore, which require $\beta=0$, such $\mathcal{I}_{2}$ terms are absent from the right-hand-side of the observable (4.1), and the (limiting) result for the asymmetry involves, in such a case, only the factor $3 \operatorname{Re} \frac{\epsilon^{\prime}}{\epsilon}\left(1-\left|\mathcal{O}\left(\gamma / \Delta \Gamma\left|\bar{\eta}_{+-}\right|^{2}\right)\right|\right)$ to leading order.

The above issues are important to bear in mind in experimental derivations of the $\epsilon^{\prime}$ parameter, which in the general case require disentanglement of the (possible) decoherence and $\omega$ effects by means of a combined study of the $\phi$-factory observables described in this article and in [5].

\section{Acknowledgments}

This work has been partially supported by the Grant CICYT FPA2002-00612. N.E.M. wishes to thank the University of Valencia, Department of Theoretical Physics and IFIC 
for the hospitality during the final stages of the collaboration. A.W.-L. also thanks the University of Valencia, Department of Theoretical Physics for the hospitality and support during the early stages of this work.

\section{Appendix: Complete Formulae with $\epsilon^{\prime}$ corrections}

In this Appendix we give the $\epsilon^{\prime}$ corrections to the pertinent double-decay rates. The inclusion of such effects is accounted for by the $Y_{+-}$parts of the observables for the Kaon decays to two and three pions in (3.1).

For the two-charged-pion decay we have

$$
\mathcal{O}_{+-}=\left|X_{+-}\right|^{2}\left(\begin{array}{cc}
1 & Y_{+-} \\
Y_{+-}^{*} & \left|Y_{+-}\right|^{2}
\end{array}\right)
$$

with $X_{+-}\left\langle\pi \pi \mid K_{2}\right\rangle Y_{\pi \pi}=\frac{\left\langle\pi \pi \mid K_{1}\right\rangle}{\left\langle\pi \pi \mid K_{2}\right\rangle}$ and we remind the reader that the quantum mechanical amplitudes $\eta_{+-}=\epsilon+\epsilon^{\prime}, \eta_{00}=\epsilon-2 \epsilon^{\prime}$, should be replaced now by their barred counterparts $\bar{\eta}$ that include decoherent contributions as well. In particular, above we have used the relations 5] $R_{L}=\frac{\gamma}{\Delta \Gamma}+\left|\bar{\eta}_{+-}\right|^{2}+4 \frac{\beta}{\Delta \Gamma} \operatorname{Im}\left[\bar{\eta}_{+-} d / d^{*}-Y_{+-}\right]$and $\left|\bar{\eta}_{+-}\right| e^{i \phi_{+-}}=\epsilon_{L}^{-}+Y^{+-}$(the reader should recall that $\left.\epsilon_{L}^{-}=\epsilon_{L}-\frac{\beta}{d}\right)$.

The relevant double-decay time distribution reads then:

$$
\begin{aligned}
\mathcal{P} & \left(\pi^{+} \pi^{-}, \tau_{1} ; \pi^{+} \pi^{-}, \tau_{2}\right)=\frac{\left|X_{+-}\right|^{4}}{2}\left[R_{L}\left(e^{-\Gamma_{L} \tau_{2}-\Gamma_{S} \tau_{1}}+e^{-\Gamma_{L} \tau_{1}-\Gamma_{S} \tau_{2}}\right)\right. \\
& -2\left|\bar{\eta}_{+-}\right|^{2} \cos \left(\Delta m\left(\tau_{1}-\tau_{2}\right) e^{-(\bar{\Gamma}+\alpha-\gamma)\left(\tau_{1}+\tau_{2}\right)}\right. \\
& +\frac{4 \beta\left|\bar{\eta}_{+-}\right|}{|d|} \sin \left(\Delta m \tau_{1}+\phi_{+-}-\phi_{S W}\right) e^{-(\bar{\Gamma}+\alpha-\gamma) \tau_{1}-\Gamma_{S} \tau_{2}} \\
& +\frac{4 \beta\left|\bar{\eta}_{+-}\right|}{|d|} \sin \left(\Delta m \tau_{2}+\phi_{+-}-\phi_{S W}\right) e^{-(\bar{\Gamma}+\alpha-\gamma) \tau_{2}-\Gamma_{S} \tau_{1}} \\
& +2|\omega|\left|\bar{\eta}_{+-}\right|\left(\cos \left(\Delta m \tau_{1}+\phi_{+-}-\Omega\right) e^{-\Gamma_{S}\left(\tau_{2}\right)-(\bar{\Gamma}+\alpha-\gamma) \tau_{1}}-\cos \left(\Delta m \tau_{2}+\phi_{+-}-\Omega\right) e^{-\Gamma_{S} \tau_{1}-(\bar{\Gamma}+\alpha-\gamma) \tau_{2}}\right) \\
& \left.+\left(|\omega|^{2}-\frac{2 \gamma}{\Delta \Gamma}-8 \frac{\beta}{\Delta \Gamma} \operatorname{Im}\left[\bar{\eta}_{+-}-Y_{+-}\right]\right) e^{-\Gamma_{S}\left(\tau_{1}+\tau_{2}\right)}\right]
\end{aligned}
$$

The time Integrated distribution reads

$$
\begin{gathered}
\overline{\mathcal{P}}\left(\pi^{+} \pi^{-} ; \pi^{+} \pi^{-} ; \Delta \tau\right)=\frac{\left|X_{+-}\right|^{4}}{2}\left[R_{L} \frac{e^{-\Delta \tau \Gamma_{L}}+e^{-\Delta \tau \Gamma_{S}}}{\Gamma_{L}+\Gamma_{S}}\right. \\
-\left|\bar{\eta}_{+-}\right|^{2} \cos (\Delta m \Delta \tau) \frac{e^{-(\bar{\Gamma}+\alpha-\gamma) \Delta \tau}}{(\bar{\Gamma}+\alpha-\gamma)}
\end{gathered}
$$



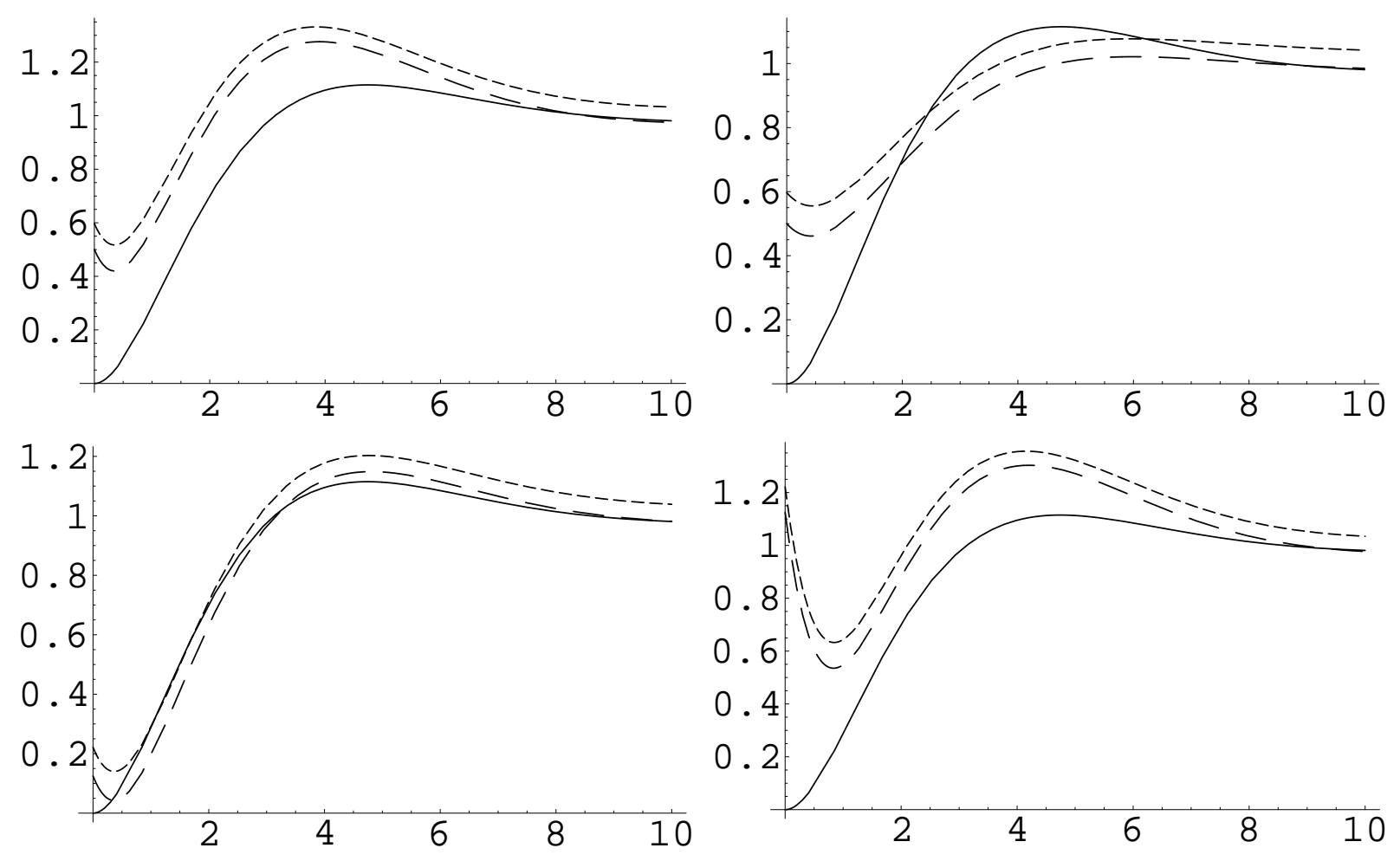

FIG. 9: Plots of $\overline{\mathcal{P}}\left(\pi^{ \pm}, \pi^{ \pm}, \Delta \tau\right)$ vs. $\Delta \tau$, including $\epsilon^{\prime}$ corrections. The long-dashed curve has only $\omega \neq 0$, whilst the solid curve represents $\alpha, \beta, \gamma, \omega=0$. The values for $\omega$ and $\Omega$ are (from left to right, and top to bottom) i) $|\omega|=\left|\bar{\eta}_{+-}\right|, \Omega=\phi_{+-}-0.16 \pi$, ii) $|\omega|=\left|\bar{\eta}_{+-}\right|, \Omega=\phi_{+-}+0.95 \pi$, iii) $\left.|\omega|=0.5\left|\bar{\eta}_{+-}\right|, \Omega=\phi_{+-}+0.16 \pi, i v\right)|\omega|=1.5\left|\bar{\eta}_{+-}\right|, \Omega=\phi_{+-} . \overline{\mathcal{P}}$ is in units of $\left|\bar{\eta}_{+-}\right|^{2} \tau_{s} 4 A_{0}^{4}$ and $\Delta \tau$ is in units of $\tau_{S}$.

$$
\begin{aligned}
& +\frac{1}{\Delta m^{2}+(\bar{\Gamma}+\alpha-\gamma)^{2}+2(\bar{\Gamma}+\alpha-\gamma) \Gamma_{S}+\Gamma_{S}^{2}} \\
& \times\left[\frac { 4 \beta | \overline { \eta } _ { + - } | } { | d | } \left(\left(\Delta m \cos \left(\phi_{+-}-\phi_{S W}\right)+\left(\bar{\Gamma}+\alpha-\gamma+\Gamma_{S}\right) \sin \left(\phi_{+-}-\phi_{S W}\right)\right) e^{-\Delta \tau \Gamma_{S}}\right.\right. \\
& +e^{-\Delta \tau(\bar{\Gamma}+\alpha-\gamma)}\left(\Delta m \cos \left(\Delta m \Delta \tau+\phi_{+-}-\phi_{S W}\right)\right. \\
& \left.\left.+\left(\bar{\Gamma}+\alpha-\gamma+\Gamma_{S}\right) \sin \left(\Delta m \Delta \tau+\phi_{+-}-\phi_{S W}\right)\right)\right) \\
& +2|\omega|\left|\bar{\eta}_{+-}\right| e^{-\Delta \tau \Gamma_{S}}\left(\left(\bar{\Gamma}+\alpha-\gamma+\Gamma_{S}\right) \cos \left(\phi_{+-}-\Omega\right)-\Delta m \sin \left(\phi_{+-}-\Omega\right)\right) \\
& -2|\omega|\left|\bar{\eta}_{+-}\right| e^{(\bar{\Gamma}+\alpha-\gamma) \Delta \tau}\left(\left(\bar{\Gamma}+\alpha-\gamma+\Gamma_{S}\right) \cos \left(\Delta m \Delta \tau+\phi_{+-}-\Omega\right)\right. \\
& \left.\left.\left.-\Delta m \sin \left(\Delta m \Delta \tau+\phi_{+-}-\Omega\right)\right)+\left(|\omega|^{2}-\frac{2 \gamma}{\Delta \Gamma}-8 \frac{\beta}{\Delta \Gamma} \operatorname{Im}\left[\bar{\eta}_{+-}-Y_{+-}\right]\right) \frac{e^{-\Gamma_{S} \Delta \tau}}{2 \Gamma_{S}}\right]\right]
\end{aligned}
$$


For the neutral-pion decay channel we use the observable:

$$
\mathcal{O}_{00}=\left|X_{00}\right|^{2}\left(\begin{array}{cc}
1 & Y_{00} \\
Y_{00}^{*} & \left|Y_{00}\right|^{2}
\end{array}\right)
$$

and the associated double-decay rate involving charged- and neutral-pion decay channels has the form:

$$
\begin{aligned}
\mathcal{P} & \left(\pi^{+} \pi^{-}, \tau_{1} ; \pi^{0} \pi^{0}, \tau_{2}\right)=\frac{\left|X_{+-}\right|^{2}\left|X_{00}\right|^{2}}{2}\left[R_{L}^{00} e^{-\Gamma_{L} \tau_{2}-\Gamma_{S} \tau_{1}}+R_{L}^{+-} e^{-\Gamma_{L} \tau_{1}-\Gamma_{S} \tau_{2}}\right. \\
& -2\left|\bar{\eta}_{+-}\right|\left|\bar{\eta}_{00}\right| \cos \left(\Delta m\left(\tau_{1}-\tau_{2}\right)+\phi_{+-}-\phi_{00}\right) e^{-(\bar{\Gamma}+\alpha-\gamma)\left(\tau_{1}+\tau_{2}\right)} \\
& +\left|\bar{\eta}_{+-}\right| e^{-\Gamma_{S} \tau_{2}-(\bar{\Gamma}+\alpha-\gamma) \tau_{1}}\left(2|\omega| \cos \left(\Delta m \tau_{1}+\phi_{+-}-\Omega\right)+\frac{4 \beta}{|d|} \sin \left(\Delta m \tau_{1}+\phi_{+-}-\phi_{S W}\right)\right) \\
& +\left|\bar{\eta}_{00}\right| e^{-\Gamma_{S} \tau_{1}-(\bar{\Gamma}+\alpha-\gamma) \tau_{2}}\left(-2|\omega| \cos \left(\Delta m \tau_{2}+\phi_{00}-\Omega\right)+\frac{4 \beta}{|d|} \sin \left(\Delta m \tau_{2}+\phi_{00}-\phi_{S W}\right)\right) \\
& \left.+\left(|\omega|^{2}-\frac{2 \gamma}{\Delta \Gamma}-4 \frac{\beta}{\Delta \Gamma} \operatorname{Im}\left[\bar{\eta}_{+-}-Y_{+-}+\bar{\eta}_{00}-Y_{00}\right]\right) e^{-\Gamma_{S}\left(\tau_{1}+\tau_{2}\right)}\right] .
\end{aligned}
$$

The double-decay time distribution involving charged-pion and dilepton channels is given by:

$$
\begin{aligned}
\mathcal{P}\left(\pi^{ \pm}, \tau_{1} ;\right. & \left.l^{ \pm}, \tau_{2}\right)=\frac{\left|X_{+-}\right|^{2}|a|^{2}}{4}\left[\left(1 \pm \delta_{L}+\frac{\gamma}{\Delta \Gamma}\right) e^{-\Gamma_{L} \tau_{2}-\Gamma_{S} \tau_{1}}+R_{L} e^{-\Gamma_{L} \tau_{1}-\Gamma_{S} \tau_{2}}\right. \\
& \mp 2\left|\bar{\eta}_{+-}\right| \cos \left(\Delta m\left(\tau_{1}-\tau_{2}\right)+\phi_{+-}\right) e^{-(\bar{\Gamma}+\alpha-\gamma)\left(\tau_{1}+\tau_{2}\right)} \\
& \pm\left(\frac{4 \beta}{|d|} \sin \left(\Delta m \tau_{2}-\phi_{S W}\right)-2|\omega| \cos \left(\Delta m \tau_{2}-\Omega\right)\right) e^{-\Gamma_{S} \tau_{1}-(\bar{\Gamma}+\alpha-\gamma) \tau_{2}} \\
& \left.+\left(|\omega|^{2}-\frac{2 \gamma}{\Delta \Gamma}-\frac{8 \beta}{\Delta \Gamma} \operatorname{Im}\left[\bar{\eta}_{+-}\right]\right) e^{-\Gamma_{S}\left(\tau_{1}+\tau_{2}\right)}\right]
\end{aligned}
$$

Finally for the three-pion decay channel the relevant observable is:

$$
\mathcal{O}_{3 \pi}=\left|X_{3 \pi}\right|^{2}\left(\begin{array}{cc}
\left|Y_{3 \pi}\right|^{2} & Y_{3 \pi}^{*} \\
Y_{3 \pi} & 1
\end{array}\right)
$$

with $\left\langle 3 \pi \mid K_{2}\right\rangle Y_{3 \pi}=\frac{\left\langle 3 \pi \mid K_{1}\right\rangle}{\left\langle 3 \pi \mid K_{2}\right\rangle}$

In the following we use $R_{S}=-\frac{\gamma}{\Delta \Gamma}+\left|\bar{\eta}_{3 \pi}\right|^{2}-4 \frac{\beta}{\Delta \Gamma} \operatorname{Im}\left[\bar{\eta}_{3 \pi} d / d^{*}-Y_{3 \pi}\right]$ where $\left|\bar{\eta}_{3 \pi}\right| e^{i \phi_{3 \pi}}=$ $\epsilon_{S}^{+}+Y_{3 \pi}$.

The pertinent three-pion double-decay time distribution is:

$\mathcal{P}\left(3 \pi, \tau_{1} ; 3 \pi, \tau_{2}\right)=\frac{\left|X_{3 \pi}\right|^{4}}{2}\left[R_{S} e^{-\Gamma_{L} \tau_{2}-\Gamma_{S} \tau_{1}}+R_{S} e^{-\Gamma_{L} \tau_{1}-\Gamma_{S} \tau_{2}}\right.$ 


$$
\begin{aligned}
& -2\left|\bar{\eta}_{3 \pi}\right|^{2} \cos \left(\Delta m\left(\tau_{1}-\tau_{2}\right)\right) e^{-(\bar{\Gamma}+\alpha-\gamma)\left(\tau_{1}+\tau_{2}\right)} \\
& +\left|\bar{\eta}_{3 \pi}\right| e^{-\Gamma_{L} \tau_{1}-(\bar{\Gamma}+\alpha-\gamma) \tau_{2}}\left(-2|\omega| \cos \left(\Delta m \tau_{2}-\phi_{3 \pi}+\Omega\right)+\frac{4 \beta}{|d|} \sin \left(\Delta m \tau_{2}-\phi_{3 \pi}+\phi_{S W}\right)\right) \\
& \left.+\left|\bar{\eta}_{3 \pi}\right| e^{-\Gamma_{L} \tau_{2}-(\bar{\Gamma}+\alpha-\gamma) \tau_{1}}\left(2|\omega| \cos \left(\Delta m \tau_{1}-\phi_{3 \pi}+\Omega\right)+\frac{4 \beta}{|d|} \sin \left(\Delta m \tau_{1}-\phi_{3 \pi}+\phi_{S W}\right)\right)\right] \\
& +\left(|\omega|^{2}+\frac{2 \gamma}{\Delta \Gamma}+\frac{8 \beta}{\Delta \Gamma} \operatorname{Im}\left[\bar{\eta}_{3 \pi}-Y_{3 \pi}\right]\right) e^{-\Gamma_{L}\left(\tau_{1}+\tau_{2}\right)}
\end{aligned}
$$

This completes our analysis. We observe that the inclusion of the $\epsilon^{\prime}$ corrections does not affect the functional form of the decoherence and CPTV effects.

[1] R.F. Streater and A.S. Wightman, PCT, Spin and Statistics, and All That, p. 207 (Redwood City, USA: Addison Wesley (1989));

[2] J. Ellis, J. Hagelin, D.V. Nanopoulos and M. Srednicki, Nucl. Phys. B241, 381 (1984).

[3] S. Hawking, Comm. Math. Phys. 43, 199 (1975); ibid. 87, 395 (1982); J. Bekenstein, Phys. Rev. D12, 3077 (1975).

[4] J. R. Ellis, N. E. Mavromatos and D. V. Nanopoulos, Phys. Lett. B 293, 142 (1992) arXiv:hep-ph/9207268; J. R. Ellis, J. L. Lopez, N. E. Mavromatos and D. V. Nanopoulos, Phys. Rev. D 53, 3846 (1996) arXiv:hep-ph/9505340;

[5] P. Huet and M. E. Peskin, Nucl. Phys. B 434, 3 (1995) arXiv:hep-ph/9403257;

[6] A. Kostelecky, arXiv:hep-ph/0403088, and references therein.

[7] G. Barenboim and J. Lykken, Phys. Lett. B 554, 73 (2003) arXiv:hep-ph/0210411.

[8] J. Bernabeu, N. E. Mavromatos and J. Papavassiliou, Phys. Rev. Lett. 92, 131601 (2004) arXiv:hep-ph/0310180.

[9] R. Wald, Phys. Rev. D21, 2742 (1980).

[10] E. Alvarez, J. Bernabeu, N. E. Mavromatos, M. Nebot and J. Papavassiliou, Phys. Lett. B 607, 197 (2005) arXiv:hep-ph/0410409.

[11] G. Lindblad, Commun. Math. Phys. 48, 119 (1976). R. Alicki and K. Lendi, Lect. Notes Phys. 286, Springer-Verlag, Berlin (1987).

[12] The authors are well aware of the limitations of a linear master equation, which may not represent the full quantum-gravity situation. The possibility that quantum gravity may be 
non-linear has already been contemplated in earlier papers (see, for instance, 19]), but any non-linearity may not be visible in a situation like neutral kaons, where a mean-field result, parametrised by a (linear) Lindblad equation, should suffice.

[13] E. Lisi, A. Marrone and D. Montanino, Phys. Rev. Lett. 85, 1166 (2000) arXiv:hep-ph/0002053; F. Benatti and R. Floreanini, JHEP 0002, 032 (2000) arXiv:hep-ph/0002221.

[14] We would like to stress at this point that such parametrizations in general may also refer to conventional decoherence effects, as a result, for instance, of the passage of the probe through ordinary matter. One therefore should be extremely careful when interpreting such effects, should the latter arise in an experimental situation. For instance, as argued in T. Ohlsson, Phys. Lett. B 502, 159 (2001), an uncertainty in the energy of the neutrino beam suffices to induce damping modifications in the respective oscillation formulae, which resemble those of decoherence. In such a case, the disentanglement of such conventional effects may come, if not from their size, which is comparable to that of QG-induced decoherence in optimistic scenaria, definitely from the details of the energy and oscillation-length dependence of the damping decoherence factors, see G. Barenboim and N. E. Mavromatos, Phys. Rev. D 70, 093015 (2004); N. E. Mavromatos, arXiv:gr-qc/0411067, For instance, ordinary matter effects mimicking CPTV decrease with the energy of the probe, in contrast to genuine QG effects..

[15] The second DAФNE Physics Handbook (Eds. L. Maiani, G. Pancheri and N. Paver, 1995).

[16] S. Eidelman et al. [Particle Data Group], Phys. Lett. B 592, 1 (2004).

[17] R. Adler et al. [CPLEAR collaboration], Phys. Lett. B 364, 239 (1995) arXiv:hep-ex/9511001.

[18] F. Benatti and R. Floreanini, Phys. Lett. B 389, 100 (1996) arXiv:hep-th/9607059; Nucl. Phys. B 511, 550 (1998) arXiv:hep-ph/9711240. Phys. Lett. B 468, 287 (1999) arXiv:hep-ph/9910508.

[19] J. R. Ellis, N. E. Mavromatos and D. V. Nanopoulos, Phys. Rev. D 63, 024024 (2001) arXiv:gr-qc/0007044.

[20] H. J. Lipkin, Phys. Rev. 176, 1715 (1968); Phys. Lett. B 219, 474 (1989); I. Dunietz, J. Hauser and J. L. Rosner, Phys. Rev. D 35, 2166 (1987);

[21] J. Bernabeu, F. J. Botella and J. Roldan, Phys. Lett. B 211, 226 (1988); Proc. 25th Anniversary of the Discovery of CP Violation, Blois, France, May 22-26, 1989, Blois CP Violations 
1989:0389-400 (QCD161:I44:1989).

[22] L. Wolfenstein, Nucl. Phys. B246, 45 (1984); M. C. Banuls and J. Bernabeu, Phys. Lett. B 464, 117 (1999); Nucl. Phys. B 590, 19 (2000).

[23] A. Apostolakis et al. [CPLEAR Collaboration], Phys. Lett. B 422, 339 (1998).

[24] G. J. Milburn, arXiv:gr-qc/0308021; see also: F. Dowker, J. Henson and R. D. Sorkin, arXiv:gr-qc/0311055. 